\title{
Spin dynamics of current-driven single magnetic adatoms and molecules
}

\author{
F. Delgado and J. Fernández-Rossier \\ Departamento de Física Aplicada, Universidad de Alicante, San Vicente del Raspeig 03690, Spain
}

(Received 29 June 2010; published 11 October 2010)

\begin{abstract}
A scanning tunneling microscope can probe the inelastic spin excitations of a single magnetic atom in a surface via spin-flip assisted tunneling in which transport electrons exchange spin and energy with the atomic spin. If the inelastic transport time, defined as the average time elapsed between two inelastic spin flip events, is shorter than the atom spin-relaxation time, the scanning tunnel microscope (STM) current can drive the spin out of equilibrium. Here we model this process using rate equations and a model Hamiltonian that describes successfully spin-flip-assisted tunneling experiments, including a single Mn atom, a Mn dimer, and Fe Phthalocyanine molecules. When the STM current is not spin polarized, the nonequilibrium spin dynamics of the magnetic atom results in nonmonotonic $d I / d V$ curves. In the case of spin-polarized STM current, the spin orientation of the magnetic atom can be controlled parallel or antiparallel to the magnetic moment of the tip. Thus, spin-polarized STM tips can be used both to probe and to control the magnetic moment of a single atom.

DOI: $10.1103 /$ PhysRevB.82.134414

PACS number(s): 72.25.Pn, 71.70.Gm, 72.25.Mk
\end{abstract}

\section{INTRODUCTION}

A single magnetic atom is arguably the smallest system where the spin can be used to store classical and/or quantum information. Therefore, there is great interest in probing and manipulating the spin state of a single atom or a single molecule in a solid-state environment. Examples of this are single phosphorous donors in silicon, ${ }^{1}$ nitrogen-vacancy centers in diamonds, ${ }^{2-5}$ single Mn atoms in II-VI (Refs. 6 and 7) and III-V (Ref. 8) semiconductors, and single magnetic adatoms in surfaces. ${ }^{9-20}$ Whereas in most cases the spin of the single atom is probed by optical means, the possibility of coupling the spin of as single atom to an electrical circuit is particularly appealing.

Tremendous recent experimental progress has made it possible to probe the spin of a single and a few atoms deposited in conducting surfaces by means of scanning tunneling microscopes. ${ }^{9-20}$ There are two complementary techniques that afford this: spin-polarized STM and spin flip inelastic electron tunnel spectroscopy (IETS). The working principle of spin-polarized STM is spin-dependent magnetoresistance, ${ }^{21}$ similar to that of tunnel magnetoresistance junction: tunneling between two spin-polarized conductors depends on the relative orientation of their magnetic moments. Control of the spin orientation of either the tip or the substrate affords spin contrast STM imaging. ${ }^{16}$

In the case of spin flip IETS, electrons tunnel from the STM tip to the surface (or vice versa), and exchange their spin with the atom, so that they produce a spin transition, whose energy is provided by the bias voltage. Thus, a new conduction channel opens when the bias voltage is larger than a given spin transition [see Figs. 1(c) and 1(d)]. This results in a step in the conductance as a function of bias and permits to determine the energy of the spin excitation and how it evolves as a function of an applied magnetic field..$^{9-13,17,18,22}$ When the atom is weakly coupled to its environment, the spin is quantized, ${ }^{23,24}$ the spin transitions have sharply defined energies which can be described with a single-ion spin Hamiltonian whose parameters can be inferred from the experiments. ${ }^{9-13}$ This is the case of $\mathrm{Mn}$, Co, and $\mathrm{Fe}$ atoms ${ }^{9-12}$ as well as $\mathrm{Fe}$ and $\mathrm{Co}$ Phthalocyanines, ${ }^{13,17,18}$ all of them deposited on a insulating monolayer on top of a metal. Remarkably, spin flip IETS does not require a spin-polarized tip to extract information about the spin dynamics.

Both IETS and spin-polarized STM are based upon the fact that the spin state of the atom affects the transport electrons, yielding a spin-dependent conductance. Therefore, we must expect that the transport electrons do affect the spin of the atom. This is the main theme of this paper. In the case of spin flip IETS, there are two relevant time scales. On one side, the inelastic transport time or charge time $T_{q}$, which is defined as the average time elapsed between two inelastic spin-flip events. On the other side, the magnetic atom spinrelaxation time, $T_{1}$. In the $T_{q} \gg T_{1}$ regime, the transport electrons always interact with an atomic spin in equilibrium with the environment. As a result, the occupation of the spin states is bias independent and the conductance is expected to have flat plateaus in between the inelastic steps. In the $T_{q} \ll T_{1}$ regime this is no longer the case. Instead, the current drives

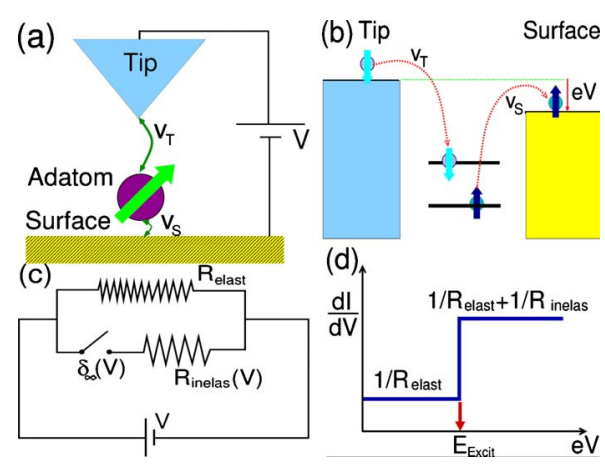

FIG. 1. (Color online) (a) Scheme of the proposed setup: a magnetic STM tip and a magnetic adatom on a insulating monolayer deposited on a metal. A current flows through the adatom when a bias voltage is applied between tip and surface. (b) Energy-level diagram of tip, atom and surface, and typical microscopic process that gives rise to spin-flip tunneling. (c) Equivalent circuit that accounts for the increase of the conductance schematically shown in (d). 
the atomic spin out of equilibrium and the occupations of the spin states are bias dependent. As we showed in a previous work, ${ }^{25}$ for the case of a single $\mathrm{Mn}$ atom, this results in a modified conductance line shape, with nonmonotonic behavior in between steps. In this work we give an extended account of these effects and consider also the case of $\mathrm{Mn}$ dimers and FePc.

Nonequilibrium effects become particularly appealing when either the tip or the substrate are also spin polarized. In this case we have current flow between two magnetic objects, which is expected to result in spin transfer torque. ${ }^{26} \mathrm{It}$ has been proposed theoretically, ${ }^{25}$ and independently verified experimentally, ${ }^{27}$ that the spin orientation of a single $\mathrm{Mn}$ atom can be controlled with a spin-polarized tip. In this paper we provide a thorough analysis of this effect and extend our study to include the effect of an external magnetic field, as in the case of the experiments. ${ }^{27}$

The results presented in this paper are based on a phenomenological spin-dependent tunneling Hamiltonian $^{22,25,28-31}$ and are, in most instances, in agreement with existing experiments. In the case of a single magnetic atom with spin $1 / 2$ it is possible to derive our spindependent Hamiltonian from a single-orbital Anderson model, by means of a Schrieffer-Wolff transformation. ${ }^{32,33}$ Within this picture, the spin-flip-assisted tunneling events would correspond to inelastic cotunneling in the Anderson model. In the single orbital Anderson model the spin-flip channel can dominate the elastic channel, which can be even zero in the so-called symmetric case. Further work ${ }^{34}$ is in progress to generalize this picture to the higher spin case relevant to this paper.

The spin dynamics of current driven nanomagnets in the Coulomb blockade regime has been thoroughly studied from the theory standpoint. ${ }^{25,35-46}$ The systems studied include magnetic grains, ${ }^{39,47}$ semiconductor ${ }^{37}$ and Mn-doped quantum dots, ${ }^{35,44}$ and molecular magnets and magnetic molecules. ${ }^{40-43,48}$ Here we can model the current driven dynamics of a quantum spin whose Hamiltonian parameters are accurately known from experiments, ${ }^{11}$ making it possible to compare successfully theory and experiment.

The rest of the manuscript is organized as follows. In Sec. II we present the model Hamiltonian for the magnetic atom(s), the transport electrons, and their coupling, which accounts both for spin-assisted tunneling and Korringa-type atomic spin relaxation due to exchange coupling with the electrodes. The transition rates and nonequilibrium dynamics leading to the current are analyzed in Sec. II C. In Sec. III we present the results of current driven spin dynamics under the influence of nonmagnetic tip in three cases: the single Mn adatom, the Mn dimer, and the FePC molecule. In Sec. IV we discuss the case of a spin-polarized tip and analyze in detail the case of a single Mn adatom. In Sec. V we present our main conclusions and discuss open questions.

\section{THEORY}

\section{A. Hamiltonian}

In this section we present the phenomenological Hamiltonian, its microscopic justification, the rate-equation ap- proach for the atom spin dynamics, including both spinrelaxation and spin-driving terms, and the calculation of the current. The system of interest is shown in Fig. 1(a). We use a model Hamiltonian which describes the system of interest split in three parts: tip, substrate, and the magnetic atom(s) ${ }^{25}$

$$
\mathcal{H}=\mathcal{H}_{\mathrm{T}}+\mathcal{H}_{\mathrm{S}}+\mathcal{H}_{\text {Spin }}+\mathcal{V}
$$

The first two terms describe the tip and surface

$$
\mathcal{H}_{\mathrm{T}}+\mathcal{H}_{\mathrm{S}}=\sum_{k, \sigma, \eta} \epsilon_{\sigma \eta}(k) c_{k \sigma \eta}^{\dagger} c_{k \sigma \eta}
$$

where $c_{k \sigma \eta}^{\dagger}$ creates and electron in electrode $\eta=T, S$, with momentum $k$ and spin $\sigma$ defined along the spin-quantization axis, $\vec{n}$. Unless stated otherwise, we take $\vec{n}$ parallel to the magnetization of the tip, which is a static vector in our theory. Since we consider a nonmagnetic surface, we have $\boldsymbol{\epsilon}_{\sigma S}(k) \equiv \boldsymbol{\epsilon}_{S}(k)$. All the results of this paper are trivially generalized to the case of a nonmagnetic tip and a magnetic surface.

The spin of the magnetic adatom(s) is (are) described with a single-ion Hamiltonian, exchange coupled to other magnetic adatoms and to the transport electrons ${ }^{10-13,22,25}$

$$
\begin{aligned}
\mathcal{H}_{\text {Spin }}= & \sum_{i}\left\{D S_{z}^{\prime 2}(i)+E\left[S_{x}^{\prime 2}(i)-S_{y}^{\prime 2}(i)\right]\right\} \\
& +\frac{1}{2} \sum_{i, j, a} J_{i, j} S_{a}^{\prime}(i) . S_{a}^{\prime}(j)+g \mu_{B} \sum_{i} \vec{S}^{\prime}(i) . \vec{B} .
\end{aligned}
$$

The first term describes the single ion magnetocrystalline anisotropy, the second describes the interatomic exchange couplings, and the third corresponds to the Zeeman splitting term under an applied magnetic field $\vec{B}$. This Hamiltonian is able to account for the excitation energies of single magnetic adatoms, ${ }^{11}$ such as $\mathrm{Mn}$ and $\mathrm{Fe}$, as well as chains of up to 10 Mn atoms deposited on $\mathrm{Cu}_{2} \mathrm{~N} .{ }^{10,22}$ The same type of Hamiltonian, combining single-ion anisotropies and interatomic exchange coupling, has been recently used to model cobalt atoms on platinum. ${ }^{20}$ Here the prime denotes that the spinquantization axis is chosen with $z^{\prime}$ along the easy axis of the system, not along the magnetic moment of the tip, $\vec{n}$. This makes necessary to rotate $\mathcal{H}_{\text {Spin }}$ when $\vec{n}$ is not parallel to the easy axis. The value of the local spin $S(i)$, the magnetic anisotropy coefficients $D$ and $E$, and the exchange coupling between atoms in the chain $J_{i, j}$, change from atom to atom and also depend on the substrate. ${ }^{10-12,22,49}$ In the following, we denote the eigenvalues and eigenvectors of $\mathcal{H}_{\text {Spin }}$ as $E_{M}$ and $|M\rangle$, respectively.

We model the coupling of the magnetic chain with the reservoirs with the following Kondo-type Hamiltonian $^{22,25,28,29,31,38}$

$$
\mathcal{V}=\sum_{\alpha, \lambda, \lambda^{\prime}, \sigma, \sigma^{\prime}, i} T_{\lambda, \lambda^{\prime}, \alpha}(i) \frac{\tau_{\sigma \sigma^{\prime}}^{(\alpha)}}{2} \hat{S}_{\alpha}(i) c_{\lambda, \sigma^{\prime}}^{\dagger} c_{\lambda^{\prime} \sigma^{\prime}},
$$

where $i$ labels the magnetic atoms in the surface, $\lambda=(k, \eta)$ labels the single-particle quantum numbers of the transport electrons (other than their spin $\sigma$ ), and the index $\alpha$ runs over four values, $a=x, y, z$, and $\alpha=0$. We use $\tau^{(a)}$ and $\hat{S}_{a}$ for the 
Pauli matrices and the spin operators in the $\vec{n}$ frame while $\hat{S}_{0}=\tau^{(0)}$ is the identity matrix. $T_{\lambda, \lambda^{\prime}, \alpha}$ for $\alpha=x, y, z$ is the exchange-tunneling interaction between the localized spin and the transport electrons, and potential scattering for $\alpha$ $=0$. Attending to the nature of the initial and final electrode, Eq. (4) describes four types of exchange interaction, two of which contribute to the current, the other two are crucial to account for the atom spin relaxation.

\section{B. Justification of the Hamiltonian}

The phenomenological spin models of Eqs. (3) and (4) capture most of the experimental results, as we show below. These models imply that the magnetic atom is in a welldefined charge state except for classically forbidden fluctuations that enable tunneling from the tip to the surface. Figure 1(b) shows a typical level alignment in which the spin model can be applied. The basic condition is therefore, that the chemical potential of the electrodes must be far enough from the chemical potential of the central-quantized region. In this way, charge addition and charge removal are classically forbidden. Although it is outside the scope of this work, we claim that the quantum charge fluctuations that give rise to spin-dependent tunneling are due to inelastic cotunneling. In the case of spin $1 / 2$, the equivalence between the spin model, originally proposed by Appelbaum ${ }^{28}$ and a single-site Anderson model was rigorously shown by Anderson ${ }^{32}$ generalizing the Schrieffer and Wolff transformation ${ }^{33}$ to the case of a single site coupled to two reservoirs. Within this picture, the atomic spin is exchanged coupled to the transport electrons and the magnitude of the exchange is given by $T_{\lambda, \lambda^{\prime}, \alpha=x, y, z}$ $\simeq \delta^{-1} V_{\lambda} V_{\lambda^{\prime}}$, where $V_{k \eta}$ is the hybridization between the Anderson site and the single-particle state $k$ in the electrode $\eta$, and $\delta$ is the energy difference between the Anderson level and the electrode Fermi energy. This is the so called Kinetic exchange. Importantly, both electrode conserving and electrode nonconserving processes are included. Their strengths are not independent since they both depend on hopping matrix elements $V_{\lambda}$ between the localized orbital in the atom and the extended orbitals in either the tip or the sample. Interestingly, the Schrieffer and Wolff transformation ${ }^{33}$ also yields a spin-independent tunneling term which would yield the $\alpha=0$ contribution in Eq. (4) and it corresponds to the elastic tunneling contribution. Within this Anderson-Kondo picture, the strength of the $\alpha=0$ elastic channel and that of the spin-dependent channel $(\alpha=x, y, z)$ are comparable and, in the so-called symmetric case, the elastic term vanishes identically. Thus, for spin- $1 / 2$ case, this picture can account for the large strength of the inelastic signal. The generalization to higher spin case, relevant for the experiments, ${ }^{9-13,27}$ will be published elsewhere. ${ }^{34}$

Keeping these considerations in mind, and following Anderson, ${ }^{32}$ we assume that Hamiltonian (4) arises from kinetic exchange. The momentum dependence of $T_{\lambda, \lambda^{\prime}, \alpha}(i)$ can have important consequences in the conductance profile ${ }^{50}$ in an energy scale of $e V$ but it can be safely neglected in IETS. We thus parametrize

$$
T_{\eta, \eta^{\prime}, \alpha}(i)=v_{\eta}(i) v_{\eta^{\prime}}(i) \mathcal{T}_{\alpha},
$$

where $v_{\mathrm{S}}(i)$ and $v_{\mathrm{T}}(i)$ are dimensionless factors that scale as the surface-adatom and tip-adatom hopping integrals. Be- cause kinetic exchange is spin-rotational invariant we have $\mathcal{T}_{x}=\mathcal{T}_{y}=\mathcal{T}_{z} \equiv|\mathcal{T}|$. Thus, Eq. (4) evinces that the spin-assisted tunneling and the atomic spin relaxations are both due to kinetic exchange, and Eq. (5) implies that their amplitudes depend on the tip-atom and surface-atom tunneling matrix elements. Furthermore, the parametrization in Eq. (5) shows that the effective coupling Hamiltonian of Eq. (4) is second order in the hopping integrals (proportional to $v_{\mathrm{S}}$ and $v_{\mathrm{T}}$ ), as it corresponds to a cotunneling process.

\section{Rates and master equation}

Our primary goal is to study transport and spin dynamics. This is done considering $\mathcal{V}$ as a perturbation to the otherwise uncoupled magnetic atom and transport electrons. The quantum spin dynamics is described by means of a master equation for the diagonal elements of the density matrix, $P_{M}$, described in the basis of eigenstates $|M\rangle$ of $\mathcal{H}_{\text {Spin }}$. The master equation is derived using the standard system plus reservoir technique ${ }^{51}$ where the transport electrons act as a reservoir for the atomic $\operatorname{spin}(\mathrm{s})$. The master equation ${ }^{51}$ reads

$$
\frac{d P_{M}}{d t}=\sum_{M} P_{M^{\prime}} W_{M^{\prime}, M}-P_{M} \sum_{M^{\prime}} W_{M, M^{\prime}},
$$

where $W_{M, M^{\prime}}$ are the transition rates between the atomic spin state $M$ and $M^{\prime}$. These rates can be written as $W_{M, M^{\prime}}$ $=\Sigma_{\eta, \eta^{\prime}} W_{M, M^{\prime}}^{\eta \rightarrow \eta^{\prime}}$, where $W_{M, M^{\prime}}^{\eta \rightarrow \eta^{\prime}}$ are the scattering rates from an atomic spin state $M$ to $M^{\prime}$ in which a quasiparticle electron goes from electrode $\eta$ to $\eta^{\prime}$ as a result of exchange process. They are given by

$$
W_{M, M^{\prime}}^{\eta \rightarrow \eta^{\prime}}=\sum_{k k^{\prime}, \sigma \sigma^{\prime}} \Gamma_{k \sigma M, k^{\prime} \sigma^{\prime} M^{\prime}}^{\eta \rightarrow \eta^{\prime}} f_{\eta}\left(\epsilon_{k \sigma}\right)\left[1-f_{\eta^{\prime}}\left(\epsilon_{k^{\prime} \sigma^{\prime}}\right)\right],
$$

where $f_{\eta}(\epsilon)=1 /\left(1+\exp \left[-\beta\left(\epsilon-\mu_{\eta}\right)\right]\right)$ is the occupation probability in electrode $\eta$ for electrons in equilibrium at chemical potential $\mu_{\eta}$ and temperature $T=1 /\left(k_{B} \beta\right) . \Gamma_{k \sigma M, k^{\prime} \sigma^{\prime} M^{\prime}}^{\eta \rightarrow \eta^{\prime}}$ is the rate at which an electron in lead $\eta$ with wave number $k$ and spin $\sigma$ is scattered into a lead $\eta^{\prime}$ with wave number $k^{\prime}$ and spin $\sigma^{\prime}$ with the impurity spin undergoing a transition between states $M$ and $M^{\prime}$. Quantum rates $\Gamma$ 's are calculated at the lowest order in the electrode-chain coupling using Fermi golden rule with the perturbation given by $\mathcal{V}$ (see Appendix A for details)

$$
\begin{aligned}
\Gamma_{k \sigma M, k^{\prime} \sigma^{\prime} M^{\prime}}^{\eta \rightarrow \eta^{\prime}}= & \frac{2 \pi}{\hbar}\left|\sum_{\alpha, i} T_{\alpha} v_{\eta}(i) v_{\eta^{\prime}}(i) S_{\alpha}^{M, M^{\prime}}(i)\right|^{2} \times \delta\left(\epsilon_{\sigma \eta}(k)\right. \\
& \left.+E_{M}-\epsilon_{\sigma^{\prime} \eta^{\prime}}\left(k^{\prime}\right)-E_{M^{\prime}}\right)
\end{aligned}
$$

where we have defined the matrix elements

$$
S_{\alpha}^{M, M^{\prime}}(i)=\left\langle M\left|S_{\alpha}(i)\right| M^{\prime}\right\rangle .
$$

The rates in Eq. (7) describe three types of processes: (1) elastic processes, $W_{M, M}^{\eta \rightarrow \eta^{\prime}}$, in which the state of atomic spin remains unchanged and a transport electron is transferred from one electrode to another. These processes are responsible for the elastic current and have no effect on the spin 
dynamics. The rates of the elastic processes scale with $v_{T}^{2} v_{S}^{2} T_{0}^{2}$. (2) Spin transitions $W_{M, M^{\prime}}^{\eta \rightarrow \eta}$. In these, a spin transition in the atomic spin is produced due to the creation or annihilation of an electron hole pair either in the tip or in the surface. These processes do not contribute to the current. At very small temperature, the fastest process of this type is atomic spin relaxation: a spin transition from an excited state $E_{M}$ to a lower energy state $E_{M^{\prime}}$ which results in the excitation of an electron hole pair in one of the electrodes. This spin-relaxation process is very similar to the nuclear spin relaxation due to hyperfine coupling to conduction electrons in metals and to $\mathrm{Mn}$ spin relaxation in diluted magnetic semiconductors due to itinerant carriers. ${ }^{7}$ At zero bias these processes dominate the atomic spin-relaxation time $T_{1}$. The rates of the spin-transition processes scale like $v_{T}^{4} \mathcal{T}^{2}$ and $v_{S}^{4} \mathcal{T}^{2}$. (3) Spin-flip-assisted tunneling $W_{M, M^{\prime}}^{\eta \rightarrow \eta^{\prime}}$. In these processes, which contribute both to inelastic current and to the dynamics of the atomic spin, a transport electron goes from electrode $\eta$ to $\eta^{\prime}$ inducing a spin transition from state $M$ to $M^{\prime}$. The rates of the spin flip assisted tunneling processes scale like $v_{T}^{2} v_{S}^{2} \mathcal{T}^{2}$.

The steady-state solutions of Eq. (6) depend, in general, on the Hamiltonian parameters, the temperature, and the bias voltage. We refer to the steady-state solutions as $P_{M}(V)$. At zero bias, the steady-state solutions are those of thermal equilibrium. At finite bias, the $P_{M}(V)$ can depart significantly from equilibrium depending on the relative efficiency of the transport-assisted spin excitations and the spin relaxation. Equation (6) does not include spin coherences. This approximation is good if the spin decoherence is faster than spin relaxation, which is known to be the case due to hyperfine coupling $^{52}$ in Mn atom. However, future work should address this point more carefully, in particular, when the magnetization of the tip $\vec{n}$ is not parallel to the single ion easy axis.

\section{Relevant parameters}

The behavior of the system is characterized by the rates in Eq. (7), which depend on a number of physical quantities like the temperature, the bias voltage $V$, density of states at the Fermi Energy of tip and surface, $\rho_{T}$ and $\rho_{S}$, the tip-atom $v_{T}(i)$ and surface-atom $v_{S}(i)$ hoppings, the spin independent $T_{0}$, and spin-dependent $\mathcal{T}$ couplings. In this work we attempt to group the unknown parameters either in terms of dimensionless numbers or as experimentally accessible quantities. For that matter, we define the zero-bias elastic conductance

$$
g_{0} \equiv \frac{\pi^{2}}{4} G_{0} \rho_{T} \rho_{S}\left|T_{0}\right|^{2} \chi^{2},
$$

where $G_{0}=2 e^{2} / h$ is the quantum of conductance and

$$
\chi=\sum_{i} v_{T}(i) v_{S}(i)
$$

is a parameter that quantifies the tip-surface transmission through the magnetic atoms. The density of states at the Fermi energy for spin $\sigma$ in the electrode $\eta$ are denoted by $\rho_{\eta \sigma}$. We define the spin assisted conductance $g_{s}$ as

$$
g_{S}=\zeta^{2} g_{0},
$$

where

$$
\zeta=\frac{\mathcal{T}}{T_{0}}
$$

is the ratio of the spin-flip-assisted and elastic tunnel matrix elements. We shall use spin polarization of the tip, defined as

$$
\mathcal{P}_{T}=\frac{\rho_{T \uparrow}-\rho_{T \downarrow}}{\rho_{T \uparrow}+\rho_{T \downarrow}} .
$$

Another important parameter is the ratio

$$
r(i) \equiv \frac{v_{T}(i)}{v_{S}(i)},
$$

which decreases as the tip is retracted from the surface. In most instances, we shall have $r(i) \leq 1$. As a general rule, the processes that drive the magnetic adatom out of equilibrium are proportional to $v_{\mathrm{T}}^{2} v_{\mathrm{S}}^{2}$ whereas the processes that cool the spin down (if $k_{b} T<|e V|$ ) are proportional to $v_{\mathrm{T}}^{4}+v_{\mathrm{S}}^{4}$. Thus, for a fixed coupling to the surface $v_{S}$, the nonequilibrium effects are higher as $r^{2} /\left(1+r^{4}\right)$ increases, reaching its maximum at $r=1$. Experimentally, $r$ can be increased moving the tip closer to the surface, ${ }^{27}$ increasing the current for a fixed applied bias $V$. In contrast, the inelastic ratio $\zeta$ and the magnitude of the tip polarization $\mathcal{P}_{T}$ are not so easy to control.

\section{E. Current}

The calculation of the rates for a tunnel event in which a transport electron goes from one electrode to the other, inducing a spin transition between states $M$ and $M^{\prime}$ (where $M$ could be equal to $M^{\prime}$ in the elastic channel), permits to obtain an expression for the current in terms of the steady state solutions of the master equation

$$
I_{S \rightarrow T}=e \sum_{M M^{\prime}} P_{M}(V)\left(W_{M, M^{\prime}}^{S \rightarrow T}-W_{M, M^{\prime}}^{T \rightarrow S}\right),
$$

where $W_{M, M^{\prime}}^{\eta \rightarrow \eta^{\prime}}$ are the scattering rates from state $M$ to $M^{\prime}$ induced by interaction with a quasiparticle which is initially in reservoir $\eta$ and ends up in $\eta^{\prime}$, given in Eq. (7). We adopt the convention that positive bias voltage $V>0$ means electrons flowing from tip to surface, see Fig. 1(b). Thus, we have

$$
e V=\mu_{\mathrm{S}}-\mu_{\mathrm{T}}
$$

with $e$ the value of the electron charge with its sign.

\section{Current for nonmagnetic tips}

The expression for the current in the case on nonmagnetic tip and substrate can be written as the sum of two terms, elastic and inelastic, $I=I_{0}+I_{I N}$ given by the expressions ${ }^{22}$

$$
I_{0}=g_{0} V
$$

where $g_{0}$ was defined in Eq. (10) and 


$$
I_{I N}=\frac{g_{S}}{G_{0}} \sum_{M, M^{\prime}, a} i_{-}\left(\Delta_{M, M^{\prime}}+e V\right)\left|\mathbf{S}_{a, T S}^{M M^{\prime}}\right|^{2} P_{M}(V)
$$

with

$$
\mathbf{S}_{a, \eta \eta^{\prime}}^{M, M^{\prime}} \equiv \frac{1}{\chi_{i}} \sum_{i} v_{\eta}(i) v_{\eta^{\prime}}(i)\left\langle M\left|S_{a}(i)\right| M^{\prime}\right\rangle
$$

Here we have introduced the current associated to a single channel with energy $\Delta_{M, M^{\prime}}=E_{M}-E_{M^{\prime}}$ and bias voltage $V$

$$
i_{ \pm}(\Delta+e V)=\frac{G_{0}}{e}[\mathcal{G}(\Delta+e V) \pm \mathcal{G}(\Delta-e V)]
$$

with $\mathcal{G}(\omega) \equiv \omega\left(1-e^{-\beta \omega}\right)^{-1}$. The curve $i_{-}$, is odd in the bias, whereas $i_{+}$, relevant in the case of magnetic tips discussed below, is even. In contrast, $d i_{-} / d V$ is even and $d i_{+} / d V$ is odd. In the nonmagnetic tip case, the elastic current in Eq. (18) provides no information about the spin state whatsoever. In contrast, the nominally inelastic terms with $E_{M}=E_{M^{\prime}}$ contribute to the conductance even at zero bias and their contribution is proportional to the spin matrix elements which can be modulated with application of a magnetic field ${ }^{53}$ Finally, the inelastic steps in conductance arise from the $M \neq M^{\prime}$ transitions in Eq. (19) and permit to extract information about the spin-transition energies, $\Delta_{M, M^{\prime}}$ and spin-matrix elements $\mathbf{S}_{a, T S}^{M, M^{\prime}}$. The basic effects of the elastic and inelastic terms in the conductance can be understood in terms of an equivalent electric circuit schematically shown in Figs. 1(c) and 1(d). For low-enough voltage, the only channels that can conduct current are the elastic ones while the inelastic channels remain closed. In this situation the (inelastic) switch is open. When the voltage is increased such as inelastic channels are open (switch is closed), these new channels contribute to the current, leading to a smaller resistance, see Fig. 1(d).

\section{Current for magnetic tips}

In the case of a magnetic tip, the current has three contributions, $I=I_{0}+I_{M R}+I_{I N}$. This result is different from the nonmagnetic case on two counts. First, the elastic case has a magnetoresistive term, so that current is now proportional to the relative orientation of the average adatom spin and the magnetic moment in the tip $\vec{n}$

$$
I_{0}+I_{M R}=g_{0}\left[1+2 \zeta\left\langle\mathbf{S}_{z, T S}\right\rangle \mathcal{P}_{T}\right] V,
$$

where

$$
\left\langle\mathbf{S}_{z, T S}\right\rangle=\sum_{M, i} P_{M}(V) v_{T}(i) v_{S}(i)\left\langle M\left|S_{a}(i)\right| M\right\rangle
$$

is the average magnetization along the $z$ axis that we take parallel to the magnetic moment of the tip. As we show both below and in Ref. 25, both $P_{M}(V)$ and the average atom magnetization depend on voltage. Importantly, the magnetoresistive contribution to the elastic current makes it possible to track changes in the single-atom magnetization experimentally.

The second difference with the nonmagnetic tip arises in the inelastic current, which is now given by the expression $^{25,29}$

$$
\begin{aligned}
I_{I N}= & \frac{g_{S}}{G_{0}} \sum_{M, M^{\prime}}\left[i_{-}\left(\Delta_{M, M^{\prime}}+e V\right) \sum_{a}\left|\mathbf{S}_{a, T S}^{M, M^{\prime}}\right|^{2}+\mathcal{P}_{T} i_{+}\left(\Delta_{M, M^{\prime}}\right.\right. \\
& \left.+e V) \Xi_{x y}\left(M, M^{\prime}\right)\right] P_{M}(V) .
\end{aligned}
$$

The new term in the second line involves the matrix elements

$$
\Xi_{x y}\left(M, M^{\prime}\right)=2 \operatorname{Im}\left[\mathbf{S}_{x, T S}^{M, M^{\prime}} \mathbf{S}_{y, T, S}^{M^{\prime}, M}\right] .
$$

As opposed to the standard inelastic current [Eq. (19)], which gives rise to steps of equal height for positive and negative bias, the $\mathcal{P}_{T}$-dependent term of the inelastic conductance, proportional to $i_{+}$, yields steps at the excitation energies of opposite sign as the polarity of the bias is reversed. Both the elastic and inelastic term proportional to $\mathcal{P}_{T}$ can produce a $d I / d V$ which is not an even function of bias.

\section{NONMAGNETIC TIP}

In this section we analyze the implications of a nonequilibrium population distribution when a finite bias is applied between tip and surface. These effects will be more relevant when the current through the system increases (by increasing the coupling to the electrodes). Next, we will study these effects in three different systems: the Mn monomer, Sec. III A, the Mn dimer, Sec. III B both deposited on a $\mathrm{Cu}_{2} \mathrm{~N}$ surface and the iron Phthalocyanine molecule, FePc, deposited on an oxidized $\mathrm{Cu}(1110)$ surface, Sec. III C.

\section{A. Mn monomer}

Let us consider first the case of a single $\mathrm{Mn}$ adatom in $\mathrm{Cu}_{2} \mathrm{~N}$, which has been widely studied experimentally ${ }^{10,27}$ and theoretically. ${ }^{22,29,45,54-58}$ The spin of the Mn atom in this environment is $S=5 / 2$. The parameters of the single-ion spin Hamiltonian have been determined experimentally to be $D$ $=-0.039 \mathrm{meV}, E=0.007 \mathrm{meV},{ }^{11}$ and $g=1.98 .{ }^{10}$ Since $E$ $\ll|D|$ we can limit our qualitative discussion to the case $E$ $=0$ so that the eigenstates of $\mathcal{H}_{\text {Spin }}$ are also eigenstates of $S_{z}$ (numerical simulations will be done with $E=0.007 \mathrm{meV}$ and do not change qualitatively). In the absence of applied magnetic field and at temperatures much smaller than the zerofield splitting $4|D|$, the equilibrium distribution is such that the two ground states, $S_{z}= \pm 5 / 2$, are equally likely and the average magnetization is zero. At an energy of $4|D|$ above the ground state level, we find a couple of degenerate excited states, with $S_{z}= \pm 3 / 2$. Finally, the two states with $S_{z}$ $= \pm 1 / 2$ are found at $6|D|$.

From the experiments, performed at low current, ${ }^{10}$ the experimental $d I / d V$ line shape is piecewise constant with two steps symmetrically located $e V= \pm 4 D$. This is accounted for by the equilibrium theory. ${ }^{22}$ As we show in Fig. 2 , and also in our previous work, ${ }^{25}$ nonequilibrium effects modify the $d I / d V$ line shape. In particular, the $d I / d V$ curve is not flat after the inelastic step and it has a small decay for $|e V|$ larger than the inelastic threshold. This nonequilibrium effect has been already observed experimentally. ${ }^{12,27}$ Using the same theory with a smaller tip-atom coupling (smaller 

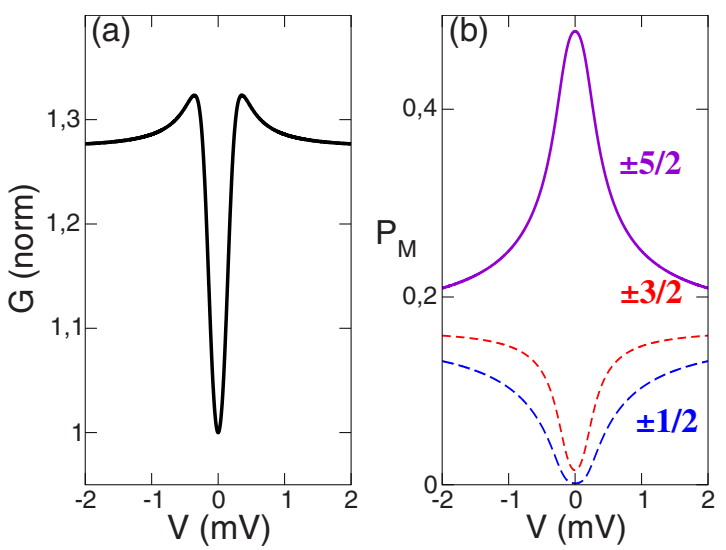

FIG. 2. (Color online) (a) $d I / d V$, normalized to the zero bias conductance, for single $\mathrm{Mn}$ in $\mathrm{Cu}_{2} \mathrm{~N}$ surface probed with a nonpolarized tip for a fixed temperature $k_{B} T=0.5 \mathrm{~K}$ and $B=0$. (b) Steadystate populations of each eigenstate versus applied bias. Here $\zeta=1$, $v_{S}=1$, and $v_{T}=1$.

$v_{T}$ ), results in $d I / d V$ line shapes identical to those equilibrium calculations. ${ }^{22}$

The nonmonotonic $d I / d V$ can be explained as follows. As the bias goes across the inelastic threshold, $|e V|=4|D|$, there is a population transfer from the ground state doublet $\left(S_{z}\right.$ $= \pm 5 / 2)$ to the first excited state doublet $S_{z}= \pm 3 / 2$. This can bee seen in Fig. 2(b). As soon as the population transfer to the first excited doublet takes place, a second inelastic channel opens: the transition from the first to the second excited state doublet $\left(S_{z}= \pm 1 / 2\right.$, whose energy is $2|D|$, smaller than the first step). It turns out the intensity of the primary inelastic step $(\Delta=4|D|)$, given by the matrix element $\left|\left\langle \pm 5 / 2\left|S^{ \pm}\right| \mp 3 / 2\right\rangle\right|^{2}$, is larger than the intensity of the secondary transition $(\Delta=2|D|)$. Thus, the depletion of the primary transition in favor of the secondary one results in a decrease in the conductance. In the case of FePc molecules, discussed below, the secondary transition is stronger than the first one, resulting in an increase of the conductance after the first step.

The nonequilibrium occupations can be understood as the balance between two driving forces. Spin-flip-assisted tunneling events heat the atomic spin, delivering energy on the order of $e V$ at a pace set by the inelastic current. The steady state is reached when the heating power is exactly compensated by dissipation. The later occurs via atomic spin relaxation due to exchange coupling to the tip and surface electrons. This process is enabled even at zero bias. Interestingly, the steady-state occupations can differ enormously from the zero bias thermal equilibrium. At $e V=2 \mathrm{meV}$, the occupation of the ground-state doublet is half of the one in equilibrium and barely twice the one of the higher energy spin levels, which are almost empty at zero bias.

The inverse of the lifetimes of the two competing processes are shown in Fig. 3. There we show the relaxation rate $1 / T_{1}$ of the magnetic spin state $S_{z}=+5 / 2$ as a function of $V$. The calculation of $T_{1}$ is done as follows. At $t=0$ we prepare the system in the state $S_{z}=+5 / 2$ and let it evolve under the dynamics defined by the master equation [Eq. (6)]. We compute the expectation value of the magnetization $\left\langle S_{z}\right\rangle(t)$ and fit
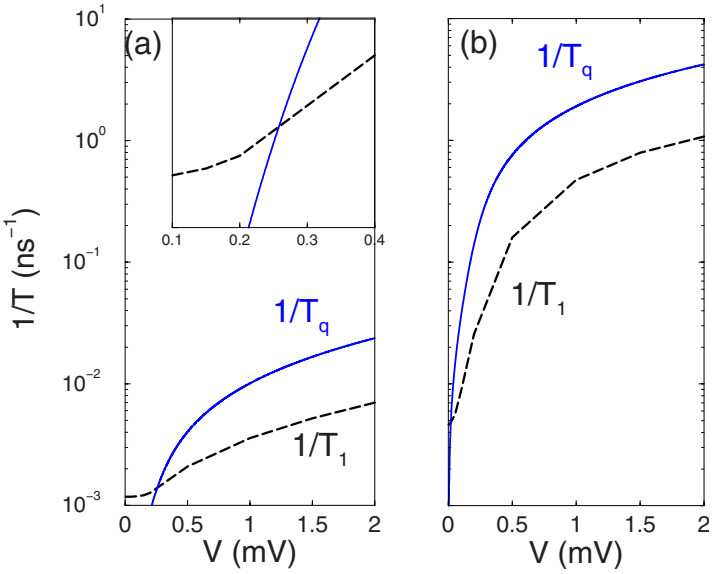

FIG. 3. (Color online) Inverse of the relaxation time of the spin state $S_{z}=+5 / 2$ (black dashed lines) and charging time $T_{q}^{-1} \equiv I_{I N} / e$ (blue solid lines), versus the applied bias for two different couplings with the tip: (a) $v_{T}=0.1$ and (b) $v_{T}=1$. In both cases, $T^{\prime}=0.5 \mathrm{~K}$, $v_{S}=1$, and $\zeta=1$. Time scales are calculated assuming zero-bias conductance $g_{0}=0.75 \mu \mathrm{S}$ for $v_{\mathrm{T}}=1$.

it to an exponentially decaying function $e^{-t / T_{1}}$ which permits to extract $T_{1}$.

When the tip is fully decoupled (no current through the system, $v_{T}=0$ ), the spin relaxes in a time scale $T_{1}$ which is independent of the applied bias. For a small coupling, Fig. $3(\mathrm{a})$, the relaxation rate increases by several orders of magnitude when the bias is increased. This effect is even more dramatic when the ratio $r$ approaches 1 [see Fig. 3(b)]. In the weak coupling curve we can easily see the crossover from the equilibrium regime at low bias, where $T_{1} \ll T_{q}$, to the nonequilibrium regime, for which $T_{1} \gg T_{q}$. In the high current case the crossover occurs at a much lower voltage.

\section{B. Dimer}

\section{Nonequilibrium effects}

From the discussion above, the nonmonotonic line shape observed for the Mn monomer is related to nonequilibrium effects. Interestingly, correlation Kondo-type effects could also modify the line shape. ${ }^{31}$ Given the fact that Kondo effect occurs in the case of a Cobalt atom deposited in the same surface,,$^{12}$ this type of effect cannot be ruled out in the Mn monomer. In contrast, the $\mathrm{Mn}$ dimer has a $S=0$ ground state ${ }^{10,22,56}$ and provides an ideal system to test the nonequilibrium physics. ${ }^{27}$

The Mn dimer was studied experimentally under lowcurrent conditions by Hirjibehedin et al. ${ }^{10}$ and, more recently, under high-current conditions by Loth et al. ${ }^{27}$ They have observed a dramatic modification of the line shape, which can be accounted for by our theory, as we show here. The Mn-Mn exchange interaction in this system is antiferromagnetic. The fitting ${ }^{10}$ of the experimental results to the Hamiltonian model, Eq. (3), gives a $J_{1,2} \equiv J=5.9$, while $D, E$ and $g$ are kept as for the monomer.

Figure 4 shows the lowest energy spectra of the Mn dimer. Since $J \gg|D|, E$, the total spin $S$ is a good quantum number at zero order in $|D| / J$. Thus, the ground state is $S$ 


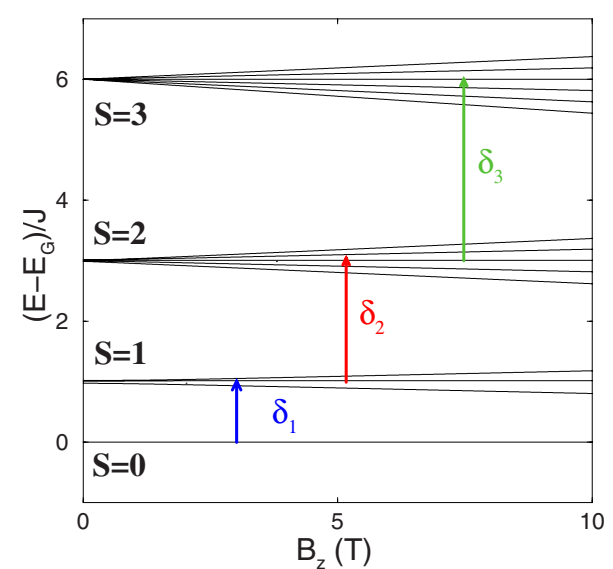

FIG. 4. (Color online) (a) Energy spectra corresponding to Hamiltonian (3) for a $\mathrm{Mn}$ dimer over a $\mathrm{Cu}_{2} \mathrm{~N}$ surface versus applied magnetic field. Spectrum is referred to the ground-state energy and given in units of the exchange coupling $(J=5.9 \mathrm{meV})$. The magnetic field is applied in the surface plane forming a 55 angle with the $\mathrm{Cu}-\mathrm{N}$ direction.

$=0$, the first excited state $S=1$ and energy $J$, the second $S$ $=2$ and energy $3 J$, and the third $S=3$ and energy $6 J$, all energies measured with respect to that of the ground state. The $2 S+1$ degeneracy of the $S>0$ multiplets is weakly lifted by the small anisotropy terms $D$ and $E$. The allowed transitions induced by the exchange coupling in Eq. (4) satisfy $\Delta S= \pm 1$. The lowest energy transitions are marked in Fig. 4 with vertical lines at energies $\delta_{i}$, with $i=1,2,3$.

The experimental results of the IETS show very different profiles as the current through the system is changed. ${ }^{27}$ For low currents only the transition at energy $\delta_{1} \approx J$ is observed and flat plateaus appear in the $d I / d V$ spectra before and after the inelastic step. This primary step corresponds to the transition from the $S=0$ ground state to the first excited state $S$ $=1$. As the current is increased, by reducing the tip-atom distance, additional steps appear at higher energies, corresponding to the transitions between the $S=1$ and $S=2$, and the $S=2$ and $S=3$ states, with energies $\delta_{2} \approx 2 J$ and $\delta_{3} \approx 3 J$. In addition, the $d I / d V$ line shapes are not flat away from the steps either. These results are reproduced by our nonequilibrium theory. Figure 5 shows the theoretical $d I / d V$ curve for three different couplings with the tip. When the tip is weakly coupled to the chain, Fig. 5(c), the step corresponding to the $S=0 \rightarrow S=1$ is clearly visible while excitations from the $S$ $=1 \rightarrow S=2$ are quenched since the $S=1$ is only slightly populated. When the coupling $v_{T}$ is increased (higher current), transitions $S=1 \rightarrow S=2$ and $S=2 \rightarrow S=3$ become possible for bias $|e V|>2 J$ and $|e V|>3 J$, respectively [see Figs. 5(b) and $5(\mathrm{a})]$. The new transitions are possible at high current due to a significant current-induced occupation of the excited states $S=1$ and $S=2$ in the Mn dimer. In contrast with the Mn monomer, the excited state spin-flip transitions energies $\delta_{2}$ and $\delta_{3}$ are larger than the primary spin transition, resulting in new steps in the spectra. These experimental results, together with the theoretical interpretation, provide strong evidence of the capability of the STM current to drive the spins of the magnetic adatoms.

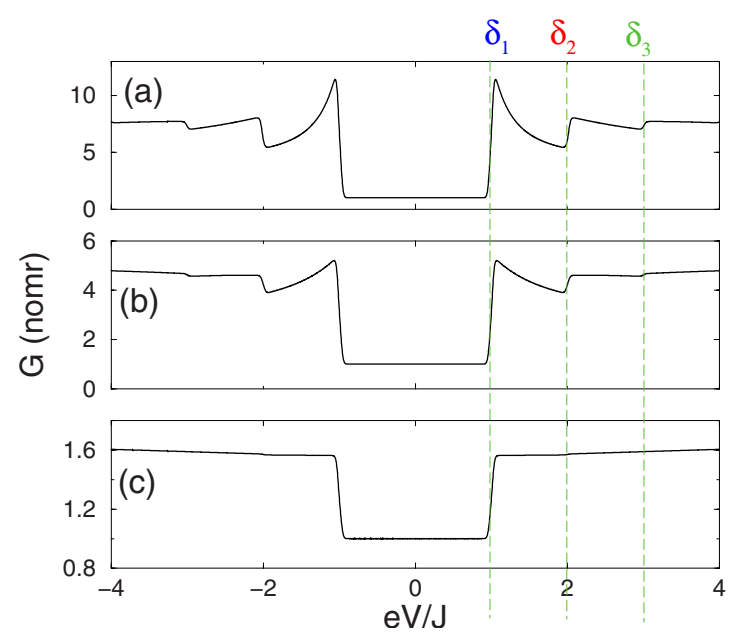

FIG. 5. (Color online) $d I / d V$ curve for the Mn dimer over a $\mathrm{Cu}_{2} \mathrm{~N}$ surface proved with a nonpolarized tip. Each panel corresponds to a pair of $\left\{v_{T}(1), v_{T}(2)\right\}$ values: (a) $\{2,1.2\}$, (b) $\{0.4,0.1\}$ and (c) $\{0.01,0.001\} . T=0.6 \mathrm{~K}, \zeta=1$, and $v_{S}(i)=0.25$.

\section{Case of symmetric coupling}

Whereas the results above are in very good agreement with the experimental data, ${ }^{27}$ it is worth pointing out that this is only so if we assume that the exchange assisted tunneling is stronger through one of the atoms. However, it vanishes identically in the symmetric coupling case, $v_{\mathrm{T}}(1)=v_{\mathrm{T}}(2)$. In Fig. 6 we plot the height of the inelastic step, given by $A_{S \rightarrow T}=\Sigma_{a} \Sigma_{M^{\prime}}\left|\mathbf{S}_{a, T S}^{G, M^{\prime}}\right|^{2}$, as a function of the lateral position of the tip across the dimer axis, as modeled by the ratio $v_{\mathrm{T}}(1) / v_{\mathrm{T}}(2)$. The inelastic step cancels identically when the tip is in the middle. This prediction of the model is at odds with unpublished experimental data, which do not show a strong dependence of the inelastic current as the tip is moved along the Mn dimer axis. From the theoretical point of view, the cancellation of the exchange assisted tunneling in the case of the Mn dimer symmetrically coupled to the tip arises from the fact that, in this particular case, the operator in the transition matrix element in Eq. (20) is the total spin of the

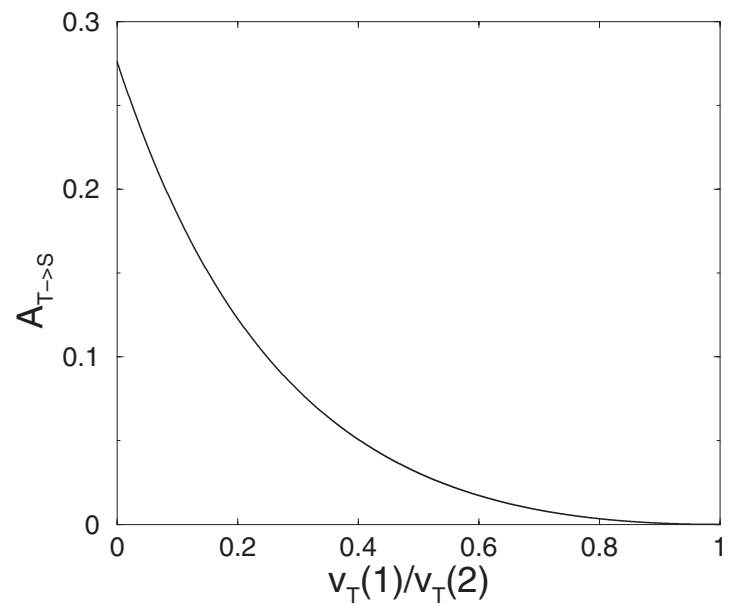

FIG. 6. Transition amplitude $A_{S \rightarrow T}$ versus the ratio $v_{\mathrm{T}}(1) / v_{\mathrm{T}}(2)$. $T=0.6 \mathrm{~K}, \zeta=1$, and $v_{S}(i)=0.25$. 
dimer, and then the eigenstates of $S^{2}$ and $S_{z}$ are also eigenstates of $\mathcal{V}$. As a result, the coupling Hamiltonian is diagonal, and no transitions are possible. Notice that this problem is specific of the dimer. In the case of the monomer the observed spin transitions occur within states with the same $S$ $=5 / 2$. In the case of the trimer and longer chains the tip cannot be coupled identically to all the atoms and the theory accounts for the data. ${ }^{22}$

There are several spin interactions other than the interatomic exchange that break the spin rotational invariance and could, in principle, solve the problem: the single ion anisotropy terms, $D$ and $E$, the hyperfine coupling with the nuclear spin of the Mn, $I=5 / 2$, and the direct magnetic dipolar coupling. We have included them in our calculations, but they are much weaker than the dominant exchange, so that they do not change qualitatively the curve of Fig. 6. Thus, even in spite of the apparent success of the perturbative approach using Eq. (4), this particular result indicates the presence of additional terms in the Hamiltonian or the need to go beyond lowest order in perturbation theory. Further work, going beyond the phenomenological theory is under way. ${ }^{34}$

\section{Magnetic molecules}

As a final example of our nonequilibrium theory with nonmagnetic electrodes, we consider the case of IETS through iron phthalocyanine $(\mathrm{FePc})$ molecules, deposited on oxidized $\mathrm{Cu}$ surface. ${ }^{17} \mathrm{FePc}$ are flat organic molecules with $C_{4}$ symmetry with a core made of a single $\mathrm{Fe}^{2+}$ ion surrounded by four nitrogen atoms embedded in benzene groups. In gas phase, the crystal field of the ligands is high enough to reduce the spin of $\mathrm{Fe}^{2+}$ from $S=2$ (high spin) to $S=1$ (intermediate spin). Because of the $C_{4}$ symmetry of the gas phase, the single-spin Hamiltonian of the molecule has $E=0$.

According to the IETS data, ${ }^{17}$ the symmetry is reduced when deposited on the oxidized surface. In particular, two adsorbed states $(\alpha$ and $\beta$ ) were experimentally observed with different spin excitations. ${ }^{17}$ In both cases the spin excitations of the FePc could be assigned to $S=1$ but the anisotropy parameters, determined from the experimental differential conductance curves, varied in the two cases. For the $\alpha(\beta)$ configuration, $D=-3.8 \pm 0.04 \mathrm{meV}(D=-6.9 \pm 0.04 \mathrm{meV})$, $E=1.0 \pm 0.01 \mathrm{meV}(E=2.1 \pm 0.04 \mathrm{meV})$, and $g=2.3 \pm 0.02$ $(g=2.4 \pm 0.05)$. The origin of this drastic change in anisotropy deserves further theory work.

The $S=1$ single-spin model can be solved analytically (see, for instance, appendix in Ref. 59). With $D<0$, and $E$ $=0$, the ground state would be the $S_{z}= \pm 1$ doublet with energy $-D$ below the $S_{z}=0$ excited state. At finite $E$ the groundstate doublet splits, in bonding and antibonding combination of the states $S_{z}= \pm 1$. The splitting is $2 E$. Thus, there are two spin transitions: the low energy one, with $\Delta=2 E$ and $\Delta S_{z}$ $=0$, and the high energy one, with energy $|D|+E$ and $\Delta S_{z}$ $= \pm 1$. The magnetic field along the $z$ axis competes with the $E$ induced splitting of the ground state. As $B$ increases, the $E$ induced mixing of the $S_{z}= \pm 1$ components decreases, and so it does the primary transition, which occurs via $\Delta S_{z}=0$ events.

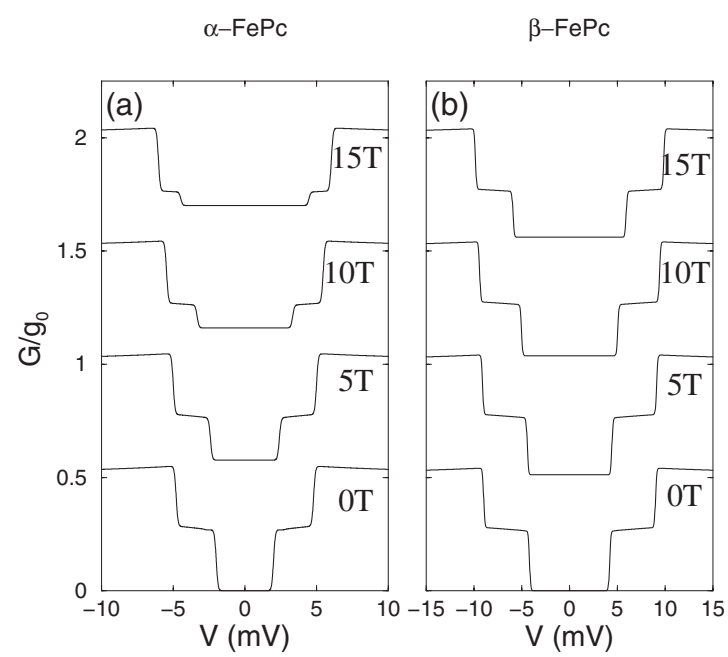

FIG. 7. Differential conductance versus applied bias at zero magnetic field for the $\alpha$ (a) and $\beta$ (b) FePc on $\mathrm{Cu}(110)(2 \times 1)-\mathrm{O}$ as a function of applied bias for different magnetic field applied perpendicular to the sample surface, as in Ref. 17. Curves have been shifted by 0.5 units for clarity. $T=0.5 \mathrm{~K}, \mathcal{P}_{T}=0, v_{S}=1, v_{T}=0.2$, and $\zeta=1$.

Figure 7 shows our nonequilibrium theoretical $d I / d V$ results using the values of $D$ and $E$ given above. Our theory reproduces not only the evolution of the steps with the magnetic field but also the mild nonequilibrium features reported in Ref. 17. After the first (second) step the conductance has a small positive (negative) slope. In contrast, the equilibrium theory ${ }^{60}$ yields flat steps. The sign of the nonequilibrium slopes depends on the relative value of the inelastic channel strengths of the primary and secondary transitions. At $\mathrm{eV}$ $=2 E$, when the first excited state is populated, the secondary transition, with energy $|D|-E$ becomes also possible at finite temperature. Since this transition between excited states has a larger quantum yield, the overall conductance increases. The opposite scenario occurs in the second step.

\section{SPIN-POLARIZED TIP}

The results of the previous section give very strong support to the notion that tunneling electrons can drive the spin of the magnetic adatoms far from equilibrium. Since we have been considering spin-unpolarized tunneling electrons, these nonequilibrium effects cannot result in a net spin transfer. From the theory standpoint, this should change dramatically in the case of spin-polarized transport electrons. As it was shown in a seminal work by Slonczewski, ${ }^{26}$ the back action of transport electrons on a magnetic moment can be used to rotate the magnetization direction. This effect, known as spin-transfer torque, have been observed in nanopillars of tens of nanometers ${ }^{61}$ down to tiny nanomagnets made of 100 atoms,${ }^{14}$ but still in the semiclassical domain. In a previous paper $^{25}$ we modeled the spin dynamics of a single Mn atom under the influence of spin-polarized current. We found that if the tip was spin polarized, the current would result in a net spin magnetization of the magnetic adatom. Its orientation, relative to the tip magnetic moment, would depend on the 

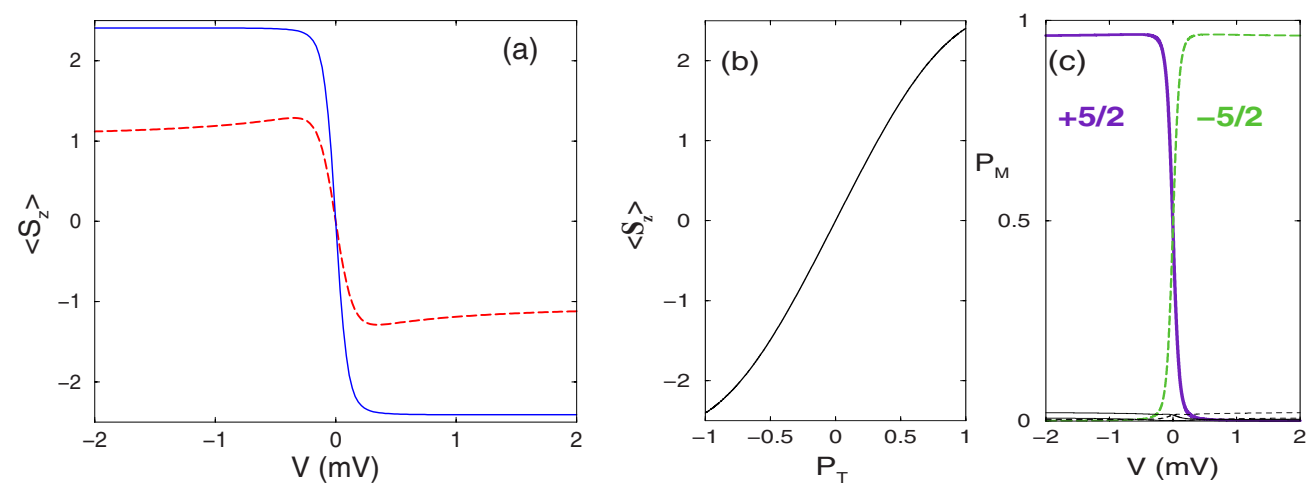

FIG. 8. (Color online) (a) Average magnetization $\left\langle S_{z}\right\rangle$ for the Mn monomer in $\mathrm{Cu}_{2} \mathrm{~N}$ surface probed with two differently polarized tips, $\mathcal{P}_{T}=-1 / 3$ (red dashed line) and $\mathcal{P}_{T}=-1$ (solid blue line) versus applied bias. (b) "Universal curve" of the magnetization versus tip polarization (for positive applied bias). (c) Steady-state populations of each eigenstate versus applied bias. Solid lines for energy levels with $S_{z}<0$ and dashed lines for $S_{z}>0$. Here $\zeta=0.5, v_{S}=1, T=0.5 \mathrm{~K}, B=0$, and $v_{T}=0.7$.

polarity of the bias, in agreement with the macroscopic spintransfer torque. In parallel to our work, Loth et al. ${ }^{27}$ demonstrated experimentally the single-atom spin transfer.

In our work in Ref. 25 the origin of the tip magnetization was ferromagnetic order. In the experiment of Loth et al., the spin-polarized current is achieved by sticking a single $\mathrm{Mn}$ atom into the tip and applying a magnetic field to freeze its spin fluctuations. The external magnetic field affects both $\mathrm{Mn}$ atoms. The very different role played by the $\mathrm{Mn}$ in the tip and the $\mathrm{Mn}$ in the surface underlines the relevance of the spin isolation. ${ }^{23,24}$ Whereas it is still possible to model the spin of the $\mathrm{Mn}$ in the surface as a quantized spin weakly coupled to the surface electrons, this picture seems to break down for the case of the Mn in the tip, due to a combination of charge transfer, Kondo coupling, and very reduced spin lifetime. Thus, we can model the experimental results of Loth et al. ${ }^{27}$ assuming that the $\mathrm{Mn}$ in the tip acts as a spin filter for the transport electrons, i.e., assuming that the tip has a finite spin polarization.

Whereas this picture works qualitatively, we believe this issue deserves further work. In the following we analyze both the case of a spin-polarized tip at zero magnetic field, and a spin-polarized tip with an external field. Only the later can be compared directly with the experiments of Loth et $a{ }^{27}$ We consider both the atomic spin dynamics under the influence of the spin-polarized current and the effect of the spin magnetization on the conductance of the system.

\section{A. Current-induced spin switching}

The flow of spin-polarized current through a single magnetic atom is expected to result in a transfer of a net spin into the atom. ${ }^{25,26}$ In the case of a single or a few magnetic atoms, where time-reversal symmetry is not spontaneously broken at zero magnetic field, the equilibrium occupation of states with opposite $S_{z}$ is the same, resulting in a null average magnetization. At finite bias, spin-polarized current changes this situation via spin-flip inelastic tunnel. ${ }^{25}$ The mechanism is the following. The dominant inelastic transitions in the case of the Mn monomer are: (a) spin increasing (SI) transition, for which the Mn spin goes from $S_{z}=-\frac{5}{2}$ to $S_{z}=-\frac{3}{2}$ and the transport electron goes from the high-energy electrode with spin $\uparrow$ to the low-energy electrode with spin $\downarrow$. (b) Spin decreasing (SD) transition, for which the Mn spin goes from $S_{z}=+\frac{5}{2}$ to $S_{z}=+\frac{3}{2}$ and the transport electron goes from the high-energy electrode with spin $\downarrow$ to the low-energy electrode with spin $\uparrow$.

In the case of spin-unpolarized current, these two processes are equally likely and result in the depletion of the two states of the ground-state doublet shown in Fig. 2(b). In the case of a spin-polarized tip, the two processes are no longer equally likely, resulting in a net spin transfer from the spin current to the atomic spin. Let us consider the case where there are more $\downarrow$ than $\uparrow$ electrons in the tip. This means negative tip magnetization (i.e., $\left.\mathcal{P}_{\mathcal{T}}<0\right)$. When electrons go from the tip to the surface $(V>0)$, the SD processes are dominant. Then the positive $S_{z}$ states are depleted and a negative $\left\langle S_{z}\right\rangle$ is expected. Thus, we expect that at positive bias (electrons going from tip to surface), the current copolarizes the spin of the atom.

We now consider electrons going from surface to tip. Since the density of states of spin $\downarrow$ electrons is higher, the SI process is now more likely than the $S T$ one. As a result, the negative $S_{z}$ states should be depleted, resulting in a positive atomic spin. Thus, we expect that for $V<0$ (electrons going from surface to tip), the current counterpolarizes the spin of the atom.

Our simulations confirm this scenario. We only consider the simplest case in which the tip polarization is parallel to the $\mathrm{Mn}$ easy axis perpendicular to the $\mathrm{Cu}_{2} \mathrm{~N}$ surface. We consider first the case of zero magnetic field. We choose $\mathcal{P}_{T}<0$, because it is convenient for the discussion at finite positive field below. In Fig. 8(a) we show the average atomic spin moment along the easy axis, as a function of the applied bias. It vanishes at zero bias, reflecting the absence of spontaneous time-reversal symmetry breaking of such a small system. At finite bias the magnetic moment aligns with the one of the tip when electrons flow from tip to surface ( $V$ $>0)$ and do exactly the opposite when the electrons flow from the surface to the tip $(V<0)$. Interestingly, the average atomic spin is finite even when $|e V|<4|D|$, the excitation energy. This is due to the existence of thermally excited quasiparticles. However, the time necessary to drive the spin of 
the atom increases exponentially when for $|e V|<4|D| .^{25}$

The average atomic spin increases both with the applied voltage and the spin polarization of the tip, $\mathcal{P}_{T}$. The effect is null at $\mathcal{P}_{T}=0$, as it should, and it is maximal for half-metallic tips $\mathcal{P}_{T}= \pm 1$. For a fixed tip polarization the effect saturates at a certain voltage. Interestingly, the saturation magnetic moment depends only on the value of $\mathcal{P}_{T}$ and is quite independent of temperature and other parameters in the calculation. We discuss this universal behavior in Sec. IV C. The nonzero atomic spin polarization reflects the bias induced asymmetry of the steady-state occupations $P_{S_{z}= \pm 5 / 2}(V)$ of the ground-state doublet $S_{z}= \pm 5 / 2$, as shown in Fig. 8(c). Notice the striking difference with the case of equilibrium, for which the occupations of these two degenerate states are identical. The steady state is reached thanks to the competition between bias induced spin transfer and exchangeinduced spin relaxation discussed in the previous section.

\section{B. Effects of spin polarization on transport}

Importantly, the current-induced polarization of the atomic spin can be detected through its influence on the conductance of the system. The simplest effect comes from the elastic magnetoresistance: conductance is larger when spin polarization of tip and magnetic atom are parallel. In the case discussed above, this results in a larger conductance at large positive bias than at large negative bias. At small bias, there are several competing effects.

Let us analyze in detail the different contributions to the conductance. For simplicity let us consider the case of a single magnetic adatom. We can write the differential conductance as

$$
G=G_{1}+G_{2}+G_{3}+G_{4},
$$

where the different terms are obtained by deriving $I$ in Eqs. (22)-(24) with respect to bias and are shown in Fig. 9(b)

$$
\begin{gathered}
G_{1}=g_{0}\left(1+2 \zeta\left\langle S_{z}\right\rangle \mathcal{P}_{T}\right), \\
G_{2}=2 g_{0} V \zeta \mathcal{P}_{T} \frac{d\left\langle S_{z}\right\rangle}{d V}, \\
G_{3}=\frac{g_{S}}{G_{0}} \sum_{M, M^{\prime}} P_{M}(V)\left[\sum_{a}\left|S_{a}^{M, M^{\prime}}\right|^{2} \times i_{-}^{\prime}\left(\Delta_{M, M^{\prime}}+e V\right)\right. \\
\left.+\mathcal{P}_{T} \Xi_{x y}\left(M, M^{\prime}\right) i_{+}^{\prime}\left(\Delta_{M, M^{\prime}}+e V\right)\right], \\
G_{4}=\frac{g_{S}}{G_{0}} \sum_{M, M^{\prime}}\left[i_{-}\left(\Delta_{M, M^{\prime}}+e V\right) \sum_{a}\left|S_{a}^{M, M^{\prime}}\right|^{2}+\mathcal{P}_{T^{i}} i_{+}\left(\Delta_{M, M^{\prime}}\right.\right. \\
\left.+e V) \Xi_{x y}\left(M, M^{\prime}\right)\right] \frac{d P_{M}(V)}{d V}
\end{gathered}
$$

with $i_{ \pm}^{\prime} \equiv d i_{ \pm} / d V . \Xi_{x y}\left(M, M^{\prime}\right)$ is defined as in Eq. (25) but with without the weighting factors. $G_{1}$ and $G_{2}\left(G_{3}\right.$ and $\left.G_{4}\right)$ correspond to the elastic (inelastic) contribution of the current. $G_{1}$ gives the dominant magnetoresistive contribution at
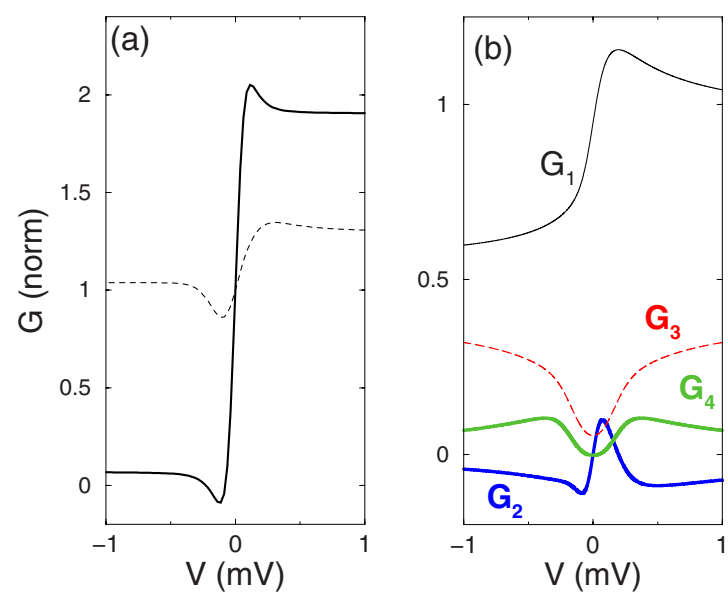

FIG. 9. (Color online) (a) Differential conductance for the Mn monomer versus the applied bias for two different tip polarizations: $\mathcal{P}_{T}=-1 / 3$ (dashed line) and $\mathcal{P}_{T}=-1$ (solid line). (b) Each of the contributions to the $d I / d V$ for $\mathcal{P}_{T}=-1 / 3: G_{1}$ (thin black line), $G_{2}$ (thick blue line), $G_{3}$ (thin dashed line) and $G_{4}$ (thick green line) versus applied bias. $T=0.5 \mathrm{~K}, \zeta=0.5$, and $v_{T}=0.7$. Magnetization direction was fix parallel to the easy axis and no magnetic field was applied.

large bias discussed above. $G_{2}$ gives a smaller contribution associated to the change of the average adatom spin as a function of bias. This term is responsible of the nonmonotonic decay of the conductance after the pronounced change induced by $G_{1}$. In the extreme case shown in Fig. 9(a), corresponding to a half metallic tip, $G_{1}$ is the dominant contribution. Finally, the two inelastic contributions, $G_{3}$ and $G_{4}$ peak close to the transition energies $\pm 4|D| . G_{3}$ corresponds to the inelastic conductance, as if the occupations $P_{M}(V)$ where bias independent, and $G_{4}$ is the contribution coming from the fact that $P_{M}(V)$ 's do depend on the bias. In turn, both $G_{3}$ and $G_{4}$ have two contributions, one that is present for nonmagnetic tip and another one proportional to the tip spin polarization $\mathcal{P}_{T}$. Importantly, the magnetic contribution to $G_{3}$ yields steps of different sign as the bias voltage is reversed.

Experimentally it might be hard to disentangle $G_{2}, G_{3}$, and $G_{4}$, but not $G_{1}$ which provides a direct way to quantify the atomic spin at large bias, denoted by $\pm V_{\infty}$

$$
\frac{G_{1}\left(+V_{\infty}\right)-G_{1}\left(-V_{\infty}\right)}{G(V=0)}=4 \zeta \mathcal{P}_{T}\left\langle S_{z}\right\rangle\left(+V_{\infty}\right),
$$

where we have used the fact that, at zero magnetic field, $\left\langle S_{z}\right\rangle\left(+V_{\infty}\right)=-\left\langle S_{z}\right\rangle\left(-V_{\infty}\right)$. Since $G_{1}$ can be the dominant contribution, replacing $G_{1}$ by the total $G$ in Eq. (28) can give a rough estimate of the quantities in the right-hand side of that equation.

\section{Finite magnetic field}

We now analyze how the magnetic field used in the experimental setup ${ }^{27}$ changes the picture discussed so far. In these experiments, the tip polarization is achieved by attaching a single $\mathrm{Mn}$ atom at the tip apex and applying a very intense magnetic field $(7 \mathrm{~T})$ perpendicular to the surface. It 


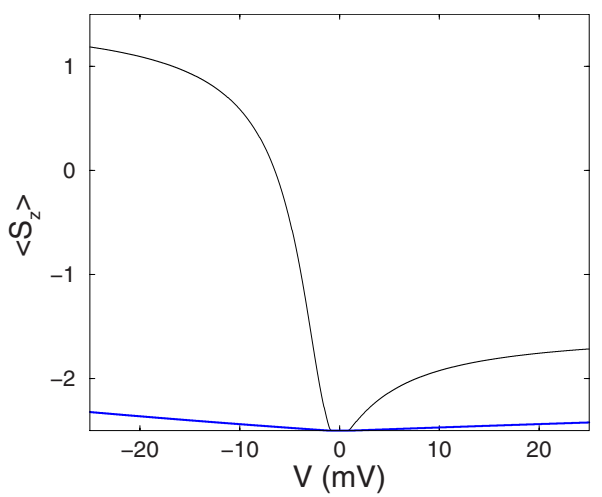

FIG. 10. (Color online) Average magnetization $\left\langle S_{z}\right\rangle$ for the Mn monomer versus applied bias for $B=7 \mathrm{~T}$ corresponding to the low current (blue line) and high current (black line) regimes. The tip magnetization direction was fix parallel to the easy axis and to the applied field. $\mathcal{P}_{T}=-0.31, T=0.5 \mathrm{~K}$, and $v_{S}=1$.

was shown ${ }^{27}$ that the $d I / d V$ curves change radically from low current to high current. In both cases, the $d I / d V$ curves are not even with respect to bias, as predicted by Delgado et $a l .{ }^{25}$ However, the line shapes differ significantly from the ones described above (Fig. 9). The origin of this discrepancy can be traced back to the effect of the magnetic field on the surface adatom, absent in our previous calculation. Since $k_{b} T \ll g \mu_{B} B$, the applied field already polarizes completely the atomic spin at zero bias. In particular, this means that the occupation of the atomic spin state $S_{z}=-5 / 2$ is very close to 1 and at zero bias, the average spin of the adatom is finite, negative, and parallel to the tip spin polarization, which maximizes the $G_{1}$ term in the conductance.

We model the two situations reported experimentally: the low-current and high-current cases. Our calculations show how, in both instances, when bias is applied, the occupation of the ground state $S_{z}=-5 / 2$ is depleted. This is shown in Fig. 10 both for the low-current case $\left(v_{T}=0.08, \zeta=0.44\right)$ and the high-current case $\left(v_{T}=0.7, \zeta=0.5\right)$. The values of $v_{T}$ and $\zeta$, as well as the value of $\mathcal{P}_{T}=-0.31$, where chosen to reproduce the experimental conductance data of Loth et al. ${ }^{27}$ In both cases the depletion of the average spin is larger for negative bias, for which transport electrons tend to counterpolarize the tip while for positive bias the depletion is associated to a nonequilibrium heating of the atomic spin. However, only in the large current case, the current is able to reverse the sign of the average atomic spin from the equilib$\operatorname{rium}\left\langle S_{z}(0)\right\rangle \equiv-2.5$ to +1 at $-20 \mathrm{mV}$, see Fig. 10 .

Now we address the question of how these bias-induced changes in the average spin can be observed in the transport experiment. In theory we can split the conductance into different contributions $G_{1}, G_{2}, G_{3}$, and $G_{4}$ for the low (Fig. 11) and high (Fig. 12) current cases, whereas in the experiment only the sum is available. In the low current case, the main contributions come from $G_{1}$, the elastic magnetoresistive term, and $G_{3}$, the inelastic conductance. The dominant $G_{1}$ contribution does not depend much on bias, since at low current the average magnetization is only weakly dependent on bias (Fig. 10). The origin of both the low bias step and the asymmetry comes mostly from the term in $G_{3}$ proportional to the tip polarization, as mentioned above. In contrast, in the
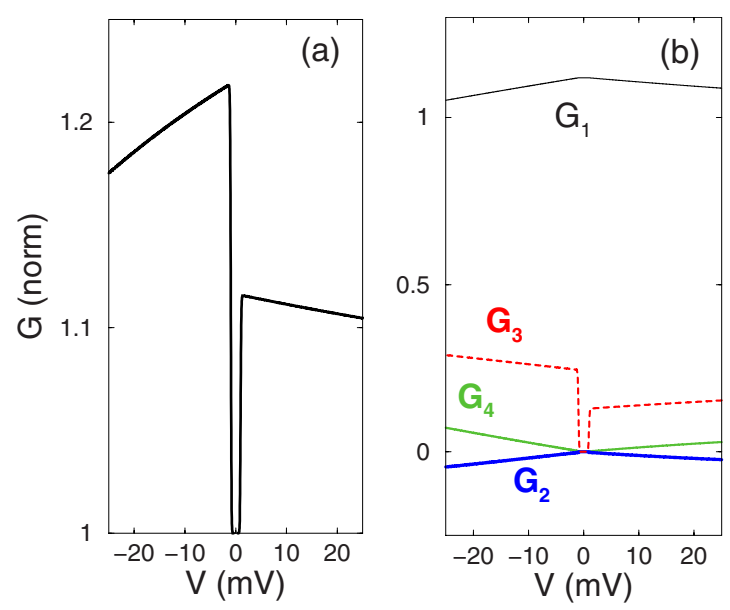

FIG. 11. (Color online) (a) Differential conductance for $B$ $=7 \mathrm{~T}$ versus applied bias for the low-current regime. (b) Each of the contributions to the $d I / d V: G_{1}$ (thin black line), $G_{2}$ (thick blue line), $G_{3}$ (thin dashed line), and $G_{2}$ (thick green line) versus applied bias. $T=0.5 \mathrm{~K}, \zeta=0.5$, and $v_{T}=0.7$. More detail in the text.

high current case, the $G_{1}$ contribution has a significant asymmetry, that arises from the fact that in this case the spin of the adatom is polarized against the tip at negative bias. This is responsible for the overall decrease in conductance observed at negative bias and backs up the notion that the magnetization of the atom can be controlled when the tip is spin polarized.

\section{Universal magnetization profile}

In Fig. 8(c) we plot the saturation magnetization, reached after a large-enough bias is applied, as a function of tip polarization. This curve turns out to be independent of all transport parameters, $\zeta, r$, and $v_{\eta} \ldots$, and it depends only on the spin of the magnetic atom $S$ and anisotropy parameters $D$ and $E$. In fact, it does not depends either on the temperature,
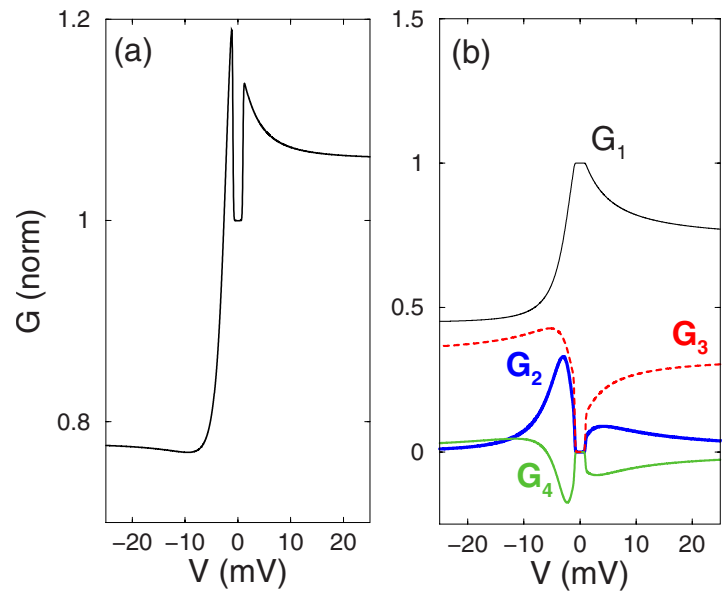

FIG. 12. (Color online) (a) Differential conductance for $B$ $=7 \mathrm{~T}$ versus applied bias for the high current regime. (b) Each of the contributions to the $d I / d V: G_{1}$ (thin black line), $G_{2}$ (thick blue line), $G_{3}$ (thin dashed line), and $G_{2}$ (thick green line) versus applied bias. $T=0.5 \mathrm{~K}, \zeta=0.5$, and $v_{T}=0.7$. More detail in the text. 
as long as our approach of neglecting the phonon contribution remains valid. When combined with Eq. (28), this could be used to determine the tip polarization.

The universality of the saturation atomic magnetization comes from the fact that, at large bias, $i_{ \pm}\left(\Delta_{M, M^{\prime}}+e V\right)$ $\approx \pm e V$ so that the Eq. (A3) for the rates can be simplified to

$$
W_{M, M^{\prime}} \propto V\left[\left|S_{z}^{M, M^{\prime}}\right|^{2}+\frac{\rho_{T \uparrow}}{\rho_{T}}\left|S_{-}^{M, M^{\prime}}\right|^{2}+\frac{\rho_{T \downarrow}}{\rho_{T}}\left|S_{+}^{M, M^{\prime}}\right|^{2}\right] .
$$

Making use of Eq. (29), the master equation for the occupation of the spin states in steady state reads

$$
\begin{aligned}
& \sum_{M^{\prime}}{ }^{\prime}\left[\left|S_{z}^{M, M^{\prime}}\right|^{2}+\frac{\mathcal{P}_{T}+1}{2}\left|S_{-}^{M, M^{\prime}}\right|^{2}+\frac{1-\mathcal{P}_{T}}{2}\left|S_{+}^{M, M^{\prime}}\right|^{2}\right] \\
& \quad \times\left(P_{M^{\prime}}-P_{M}\right)=0, \quad \forall M,
\end{aligned}
$$

where the prime indicates sum is done over $M^{\prime} \neq M$. Equation (30) shows that, in the large bias limit, the atomic spin steady-state occupations $P_{M}$, and consequently the average magnetization $\left\langle S_{z}\right\rangle$, depend only on the matrix elements of the spin operators and the polarization of the tip, and do not depend on the coupling strength to the tip and surface.

\section{SUMMARY AND CONCLUSIONS}

We have studied the mutual influence of transport electrons and the spin of localized magnetic impurities in STM configuration. Our results indicate that nonequilibrium effects are essential to understand present IETS STM spectra of magnetic adatoms. We are able to describe correctly the experimental observations of IETS on single Mn atoms, both with nonmagnetic ${ }^{11}$ and magnetic ${ }^{27}$ tips, on $\mathrm{Mn}$ dimers at low and high current, ${ }^{27}$ and on FePc molecules. ${ }^{17}$

Whereas nonmonotonic $d I / d V$ had been already observed experimentally, the recent results reported by Loth et al. ${ }^{27}$ have confirmed this scenario by controlling the tip-adatom distance. In addition, the use of spin-polarized tips amplifies the changes in the $d I / d V$ curves as the conductance is increased. The results of Loth et al. ${ }^{27}$ indicate that, in the case of magnetic tips, the orientation of the average atomic spin can be switched at will from parallel $(V>0)$ to antiparallel $(V<0)$ with respect to the magnetic tip. The control of the spin of a single atom and a single magnetic molecule had been predicted by theory. ${ }^{22,48}$ The control of atomic spin with nonequilibrium spin-polarized carriers is similar to that obtained by optical pumping. ${ }^{52}$

The main conclusions are the following: (1) the dynamics of the atomic spin under the influence of tunneling electrons is governed by two intrinsic time scales, the inelastic transport time $T_{q}$ and the atomic spin-relaxation time $T_{1}$. When the current induced spin flips occur more often than the time it takes to the atomic spin to relax, i.e., when $T_{q}<T_{1}$, nonequilibrium effects buildup. This makes the occupation of the spin states different from that of equilibrium. (2) The conductance line shape is sensitive to the occupation of the atomic spin states. This might be used to perform transportdetected single-atom resonance experiments. Our calcula- tions indicate that nonequilibrium effects have been observed in single Mn atoms, in Mn dimers and in FePc molecules. (3) A rough estimate of the quality factor of the spin excitation with energy $\Delta=\hbar \omega$, defined as $Q=\omega T_{1}$, can be obtained from the transport experiments. We assume that the current at which the low temperature conductance line shape starts to deviate from a piecewise constant function yields $T_{q} \simeq T_{1}$. Let $\Delta G_{\text {in }}$ and $V=\frac{\hbar \omega}{e}$ be the height and bias of the primary inelastic step. Then, the inelastic current is $I_{\mathrm{IN}}=\Delta G_{\text {in }} V$ $=e / T_{q}$. Then we get

$$
\omega T_{1} \simeq \frac{G_{0}}{\Delta G_{\mathrm{in}}}
$$

(4) Spin-polarized STM can be used to magnetize the atomic spin both parallel or antiparallel to the magnetic tip moment. When electrons tunnel through the magnetic atom from the magnetic tip to the surface, the atomic spin is magnetized parallel to the tip. Reversing the bias results in an opposite spin polarization. (5) The bias-induced adatom spinpolarization results in asymmetric conductance line shapes due, in most part, to the dependence of conductance on the relative orientation of the adatom and tip magnetizations. (6) The saturation atomic spin magnetization, obtained at large bias, is only a function of the impurity spin, the anisotropy parameters and the polarization of the tip.

Future work should address open problems, such as the origin of the spin assisted tunneling Hamiltonian for spin larger than $1 / 2$, the effect of atomic spin coherence, which we have neglected in the master Eq. (6) and the fact that the observed inelastic steps in the Mn dimer do not depend on the lateral position of the tip, in contrast with our theory. Recently, we became aware of a complementary work by Novaes et al. ${ }^{62}$

\section{ACKNOWLEDGMENTS}

We acknowledge fruitful discussions with A. S. Núñez, J. J. Palacios, C. F. Hirjibehedin, and S. Loth. This work has been financially supported by MEC-Spain (Grants No. JCI2008-01885, No. MAT07-67845, and CONSOLIDER No. CSD2007-00010).

\section{APPENDIX A: EQUATIONS FOR THE RATES}

In this appendix we derive the general expression of the transition rates and we work out analytical expressions in some simple cases. Applying the Fermi golden rule using the tunneling Hamiltonian (4) as perturbation, one gets

$$
\begin{aligned}
\Gamma_{k \sigma M, k^{\prime} \sigma^{\prime} M^{\prime}}^{\eta \rightarrow \eta^{\prime}}= & \frac{2 \pi}{\hbar} \delta\left(\epsilon_{\sigma \eta}(k)+E_{M}-\epsilon_{\sigma^{\prime} \eta^{\prime}}\left(k^{\prime}\right)-E_{M^{\prime}}\right) \\
& \times\left|\sum_{\alpha, \sigma \sigma^{\prime}} T_{\alpha} \frac{\tau_{\sigma \sigma^{\prime}}^{(\alpha)}}{2} \mathbf{S}_{\alpha, \eta \eta^{\prime}}^{M, M^{\prime}}\right|^{2} .
\end{aligned}
$$

The modulus square in Eq. (A1) can be expanded as 


$$
\sum_{\alpha, \beta, \sigma \sigma^{\prime}} T_{\alpha} T_{\beta} \frac{\tau_{\sigma \sigma^{\prime}}^{(\alpha)}}{2} \frac{\tau_{\sigma^{\prime} \sigma}^{(\beta)}}{2} \mathbf{S}_{\alpha, \eta \eta^{\prime}}^{M, M^{\prime}} \mathbf{S}_{\beta, \eta \eta^{\prime}}^{M^{\prime}, M}
$$

Considering the explicit form of the Pauli matrix elements, the sum over $\sigma$ and $\sigma^{\prime}$ can be done, with just a few nonzero contributions. Using the definition of the transition rates $W_{M, M^{\prime}}^{\eta \rightarrow \eta^{\prime}}$, Eq. (7), we obtain after some algebra

$$
W_{M, M^{\prime}}^{\eta \rightarrow \eta^{\prime}}=\frac{2 \pi T_{0}^{2} \chi^{2}}{\hbar} \mathcal{G}\left(\Delta_{M, M^{\prime}}+\mu_{\eta}-\mu_{\eta^{\prime}}\right) \Sigma_{M, M^{\prime}}^{\eta \eta^{\prime}},
$$

where

$$
\begin{aligned}
\sum_{M, M^{\prime}}^{\eta \eta^{\prime}}= & \frac{1}{4} \delta_{M M^{\prime}}\left[\mathcal{R}^{+}\left(\eta \eta^{\prime}\right)+2 \zeta \mathcal{R}^{-}\left(\eta \eta^{\prime}\right) \sum_{a} \mathbf{S}_{a, \eta \eta^{\prime}}^{M, M}\right] \\
& +\zeta^{2}\left[\left|\mathbf{S}_{+, \eta \eta^{\prime}}^{M, M^{\prime}}\right|^{2} \rho_{\eta \downarrow} \rho_{\eta^{\prime} \uparrow}+\left|\mathbf{S}_{-, \eta \eta^{\prime}}^{M, M^{\prime}}\right|^{2} \rho_{\eta \uparrow} \rho_{\eta^{\prime} \downarrow}+\mathcal{R}^{+}\left(\eta \eta^{\prime}\right)\right. \\
& \left.\times\left|\mathbf{S}_{z, \eta \eta^{\prime}}^{M, M^{\prime}}\right|^{2}\right]
\end{aligned}
$$

Here we have introduced $\mathcal{R}^{ \pm}\left(\eta \eta^{\prime}\right)=\rho_{\eta \uparrow} \rho_{\eta^{\prime} \uparrow} \pm \rho_{\eta \downarrow} \rho_{\eta^{\prime} \downarrow}$ and the operators $\mathbf{S}_{ \pm}=\mathbf{S}_{x} \pm i \mathbf{S}_{y}$. Notice that expression (A4) includes both terms where $M=M^{\prime}$, relevant in the calculation of the elastic contribution of the conductance, and scattering terms with $M \neq M^{\prime}$.

We now expand Eq. (A3) for some simple situations. We consider the case of a single magnetic adatom in a spinunpolarized case so that $\rho_{S}=2 \rho_{S, \uparrow}=2 \rho_{S, \downarrow}$ and $\rho_{T}=2 \rho_{T, \uparrow}$ $=2 \rho_{T, \downarrow}$. We consider only transitions with $M \neq M^{\prime}$. Combining Eqs. (9), (11), (13), (20), (A3), and (A4), the transition rate for the single atomic spin to go from state $M$ to state $M^{\prime}$ due to the excitation of an electron-hole pair in the surface reads

$$
W_{M, M^{\prime}}^{S \rightarrow S}=\frac{2 \pi T^{2}}{\hbar} v_{S}^{4} \mathcal{G}(\Delta) \frac{\rho_{S}^{2}}{4} \sum_{a=x, y, z}\left|S_{a}^{M, M^{\prime}}\right|^{2},
$$

where $\Delta=\Delta_{M, M^{\prime}}$ is the energy difference between the initial $(M)$ and final $\left(M^{\prime}\right)$ state. Notice how the rate scale with the square of the spin-flip exchange coupling $T$, and with the fourth power of the dimensionless scaling factor $v_{S}$. The $\mathcal{G}$ function can be written as

$$
\mathcal{G}(\Delta)=\frac{\Delta}{1-e^{-\beta \Delta}}=\Delta\left[1+n_{B}(\Delta)\right]
$$

where $n_{B}$ is the Bose occupation function. In the case of a cooling transition with $\Delta>0$ this equation has a transparent interpretation: the rate has a contribution coming from the spontaneous emission of an electron hole pair plus a second contribution, proportional to $n_{B}(\Delta)$, associated to the stimulated emission. The former is always possible whereas the later is thermally activated. In the case of heating transitions, for which the final spin state has higher energy than the initial state and $\Delta<0$ it helps to define $\Omega \equiv-\Delta$ and write

$$
\mathcal{G}(-|\Delta|)=\mathcal{G}(\Omega)=\Omega n_{B}(\Omega) .
$$

Here $\Omega$ is the energy released in the atomic spin by the annihilation of a thermally excited electron hole pair in the surface. The rate is proportional to the occupation of such excitation. For $k_{b} T \ll \Omega$ we can approximate $\mathcal{G}(\Omega) \simeq \Omega e^{-\beta \Omega}$, i.e., the process is very unlikely. In the opposite limit, $k_{B} T$ $\gg \Omega$ we can approximate $\mathcal{G}(\Omega) \simeq k_{B} T$, i.e, the process is thermally activated.

The rate $W_{M, M^{\prime}}^{T \rightarrow T}$ associated to the creation of an electronhole pair in the tip is obtained replacing $v_{S}$ by $v_{T}$ and $\rho_{S}$ by $\rho_{T}$. These two processes, $W_{M, M^{\prime}}^{T \rightarrow T}$ and $W_{M, M^{\prime}}^{S \rightarrow S}$ are the main contributors to the relaxation of the atomic spin, and therefore of $T_{1}$. However, they do not contribute to the current, which requires the scattering of an electron from the tip to the surface (or vice versa). The corresponding rate reads

$$
W_{M, M^{\prime}}^{T \rightarrow S}=\frac{2 \pi T^{2} v_{S}^{2} v_{T}^{2}}{\hbar} \mathcal{G}(\Delta-e V) \frac{\rho_{S}}{4} \rho_{T} \sum_{a}\left|S_{a}^{M, M^{\prime}}\right|^{2} .
$$

This rate governs the spin-flip-assisted tunnel current and scales with the square of the matrix elements $\left|S_{a}^{M, M^{\prime}}\right|^{2}$, as observed experimentally. ${ }^{11}$ In the case of the single-spin Hamiltonian with uniaxial anisotropy, $\mathcal{H}=D S_{z}^{2}$ it is trivial to obtain analytical expressions for $\sum_{a=x, y, z}\left|S_{a}^{M, M^{\prime}}\right|^{2}$. In this case we can label the eigenstates of $\mathcal{H}$ with $m$, the eigenvalues of $S_{z}$. Transitions between states with different $m$ occur only through the $a=x, y$ channels and have the selection rule $m$ $=m^{\prime} \pm 1$

$$
\sum_{a}\left|S_{a}^{m, m^{\prime}}\right|^{2}=\frac{S(S+1)-m m^{\prime}}{2}\left(\delta_{m, m^{\prime}+1}+\delta_{m+1, m^{\prime}}\right),
$$

where $S$ is the total spin.

It is possible and convenient to relate the rates in Eqs. (A5) and (A8) with the experimental conductance. In particular, using the definition of the zero bias elastic conductance in Eqs. (10) and (12), we can write

$$
\hbar W_{M, M^{\prime}}^{T \rightarrow S}=\frac{8}{\pi} \frac{g_{s}}{G_{0}} \mathcal{G}(\Delta-e V) \sum_{a=x, y, z}\left|S_{a}^{M, M^{\prime}}\right|^{2}
$$

and using Eq. (15)

$$
\hbar W_{M, M^{\prime}}^{S \rightarrow S}=\frac{8}{\pi r^{2}} \frac{g_{S}}{G_{0}} \mathcal{G}(\Delta) \frac{\rho_{S}}{\rho_{T a=x, y, z}} \sum_{a}\left|S_{a}^{M, M^{\prime}}\right|^{2} .
$$

From these expressions we see how the rates are proportional to $g_{s}$, which can be obtained from the height of the inelastic steps in the differential conductance. The expression (A11), which can be interpreted as broadening of the spin excitation $M$ to $M^{\prime}$ due to coupling to the surface, also shows the limit of application of the theory when transport is not in the tunnel regime. In the low-temperature limit we can approximate $\mathcal{G}(\Delta)$ by $\Delta$. If we consider the case in which $\rho_{T} \simeq \rho_{S}$ and $v_{T} \simeq v_{S}$, the requirement that the broadening $\hbar W$ is much smaller than $\Delta$ is equivalent to the requirement that $g_{s}$ is much smaller than $G_{0}$, i.e., that the inelastic conductance is in the tunnel regime. The scattering rates in Eq. (A11) determine the relaxation dynamics of the spin and can be used to estimate $T_{1}$. In the simplified case of an uniaxial spin model $(E=0)$, with $D<0$ and $S=5 / 2$, the spin-relaxation rate from the first excited state to the ground state reads 


$$
\hbar W_{3 / 2,5 / 2}^{S \rightarrow S}=\frac{20}{\pi} \frac{g_{s}}{G_{0}} \Delta
$$

if we take $\rho_{s}=\rho_{T}$ and $r=1$ and the low-temperature approximation. This backs up the heuristic result of Eq. (31).

\section{APPENDIX B: EQUATIONS FOR THE CURRENT}

Here we shall derive expressions (18) and (19). Let us start with the expression for the current, Eq. (16). Dividing the contribution into its elastic and inelastic part, with the help of Eq. (A3) and (A4), we can write

$$
\begin{aligned}
I_{0}+I_{M R}= & \frac{2 \pi e T_{0}^{2}}{\hbar} \frac{\chi^{2} \rho_{S} \rho_{T}}{8}\left[1+2 \zeta\left\langle\mathbf{S}_{z, T, S}\right\rangle \mathcal{P}_{T}\right] \\
& \times[\mathcal{G}(e V)+\mathcal{G}(-e V)]
\end{aligned}
$$

or, using the definition of $g_{0}$ and $i_{+}$

$$
I_{0}+I_{M R}=\frac{g_{0}}{e}\left[1+2 \zeta\left\langle\mathbf{S}_{z, T, S}\right\rangle \mathcal{P}_{T}\right] i_{-}(e V)
$$

which is the result of Eq. (18). For the inelastic contribution, the difference $W_{M M^{\prime}}^{S \rightarrow T}-W_{M, M^{\prime}}^{T \rightarrow S}$ can be written with the help of Eqs. (A3) and (A4) as

$$
\begin{aligned}
W_{M M^{\prime}}^{T \rightarrow S}-W_{M, M^{\prime}}^{S \rightarrow T}= & \zeta^{2} \frac{2 \pi \chi^{2} \rho_{S} \rho_{T} T_{0}^{2}}{8 \hbar}\left[i_{-}\left(\Delta_{M, M^{\prime}}+e V\right)\right. \\
& \times \sum_{a}\left|\mathbf{S}_{a, T, S}^{M, M^{\prime}}\right|^{2}+\mathcal{P}_{T^{\prime}} i_{+}\left(\Delta_{M, M^{\prime}}+e V\right) \\
& \times 2 \operatorname{Im}\left[\mathbf{S}_{x, T, S}^{M, M^{\prime}} \mathbf{S}_{y, T, S}^{M^{\prime}, M}\right] .
\end{aligned}
$$

Using the definitions of $g_{s}$ and $\Xi_{x y}\left(M, M^{\prime}\right)$, expression (19) is recovered.
${ }^{1}$ B. Kane, Nature (London) 393, 133 (1998).

${ }^{2}$ F. Jelezko, T. Gaebel, I. Popa, M. Domhan, A. Gruber, and J. Wrachtrup, Phys. Rev. Lett. 93, 130501 (2004).

${ }^{3}$ L. Childress, M. V. Gurudev Dutt, J. M. Taylor, A. S. Zibrov, F. Jelezko, J. Wrachtrup, P. R. Hemmer, and M. D. Lukin, Science 314, 281 (2006).

${ }^{4}$ R. Hanson, V. V. Dobrovitski, A. E. Feiguin, O. Gywat, and D. D. Awschalom, Science 320, 352 (2008).

${ }^{5}$ P. Neumann, N. Mizuochi, F. Rempp, P. Hemmer, H. Watanabe, S. Yamasaki, V. Jacques, T. Gaebel, F. Jelezko, and J. Wrachtrup, Science 320, 1326 (2008).

${ }^{6}$ Y. Léger, L. Besombes, J. Fernández-Rossier, L. Maingault, and H. Mariette, Phys. Rev. Lett. 97, 107401 (2006).

${ }^{7}$ L. Besombes, Y. Leger, J. Bernos, H. Boukari, H. Mariette, J. P. Poizat, T. Clement, J. Fernández-Rossier, and R. Aguado, Phys. Rev. B 78, 125324 (2008).

${ }^{8}$ A. Kudelski, A. Lemaître, A. Miard, P. Voisin, T. C. M. Graham, R. J. Warburton, and O. Krebs, Phys. Rev. Lett. 99, 247209 (2007).

${ }^{9}$ A. J. Heinrich, J. A. Gupta, C. P. Lutz, and D. M. Eigler, Science 306, 466 (2004).

${ }^{10}$ C. F. Hirjibehedin, C. P. Lutz, and A. J. Heinrich, Science 312, 1021 (2006).

${ }^{11}$ C. Hirjibehedin, C.-Y. Lin, A. Otte, M. Ternes, C. P. Lutz, B. A. Jones, and A. J. Heinrich, Science 317, 1199 (2007).

${ }^{12}$ A. F. Otte, M. Ternes, K. von Bergmann, S. Loth, H. Brune, C. P. Lutz, C. F. Hirjibehedin, and A. J. Heinrich, Nat. Phys. 4, 847 (2008).

${ }^{13}$ X. Chen, Y.-S. Fu, S.-H. Ji, T. Zhang, P. Cheng, X.-C. Ma, X.-L. Zou, W.-H. Duan, J.-F. Jia, and Q.-K. Xue, Phys. Rev. Lett. 101, 197208 (2008).

${ }^{14}$ S. Krause, L. Berbil-Bautista, G. Herzog, M. Bode, and R. Wiesendanger, Science 317, 1537 (2007).

${ }^{15}$ F. Meier, L. Zhou, J. Wiebe, and R. Wiesendanger, Science 320, 82 (2008).

${ }^{16}$ R. Wiesendanger, Rev. Mod. Phys. 81, 1495 (2009).

${ }^{17}$ N. Tsukahara et al., Phys. Rev. Lett. 102, 167203 (2009).
${ }^{18}$ Y. S. Fu, T. Zhang, S. H. Ji, X. Chen, X. C. Ma, J. F. Jia, and Q. K. Xue, Phys. Rev. Lett. 103, 257202 (2009).

${ }^{19}$ H. Brune and P. Gambardella, Surf. Sci. 603, 1812 (2009).

${ }^{20}$ L. Zhou, J. Wiebe, S. Lounis, E. Vedmedenko, F. Meier, S. Blügel, P. H. Dederichs, and R. Wiesendanger, Nat. Phys. 6, 187 (2010).

${ }^{21}$ J. Slonczewski, Phys. Rev. B 39, 6995 (1989).

${ }^{22}$ J. Fernández-Rossier, Phys. Rev. Lett. 102, 256802 (2009).

${ }^{23}$ C. M. Canali and A. H. MacDonald, Phys. Rev. Lett. 85, 5623 (2000).

${ }^{24}$ T. O. Strandberg, C. M. Canali, and A. H. MacDonald, Nature Mater. 6, 648 (2007).

${ }^{25}$ F. Delgado, J. J. Palacios, and J. Fernández-Rossier, Phys. Rev. Lett. 104, 026601 (2010).

${ }^{26}$ J. Slonczewski, J. Magn. Magn. Mater. 159, L1 (1996).

${ }^{27}$ S. Loth, K. von Bergmann, M. Ternes, A. F. Otte, C. P. Lutz, and A. J. Heinrich, Nat. Phys. 6, 340 (2010).

${ }^{28}$ J. A. Appelbaum, Phys. Rev. 154, 633 (1967).

${ }^{29}$ J. Fransson, Nano Lett. 9, 2414 (2009).

${ }^{30}$ J. Fransson, O. Eriksson, and A. V. Balatsky, Phys. Rev. B 81, 115454 (2010).

${ }^{31}$ R. Zitko and T. Pruschke, New J. Phys. 12, 063040 (2010).

${ }^{32}$ P. W. Anderson, Phys. Rev. Lett. 17, 95 (1966).

${ }^{33}$ J. R. Schrieffer and P. A. Wolff, Phys. Rev. 149, 491 (1966).

${ }^{34}$ F. Delgado and J. Fernández-Rossier (unpublished).

${ }^{35}$ A. L. Efros, E. I. Rashba, and M. Rosen, Phys. Rev. Lett. 87, 206601 (2001).

${ }^{36}$ J.-i. Inoue and A. Brataas, Phys. Rev. B 70, 140406 (2004).

${ }^{37}$ M. Braun, J. König, and J. Martinek, Phys. Rev. B 70, 195345 (2004).

${ }^{38}$ G. H. Kim and T. S. Kim, Phys. Rev. Lett. 92, 137203 (2004).

${ }^{39}$ X. Waintal and O. Parcollet, Phys. Rev. Lett. 94, 247206 (2005).

${ }^{40}$ C. Timm and F. Elste, Phys. Rev. B 73, 235304 (2006).

${ }^{41}$ C. Romeike, M. R. Wegewijs, and H. Schoeller, Phys. Rev. Lett. 96, 196805 (2006).

${ }^{42}$ J. Lehmann and D. Loss, Phys. Rev. Lett. 98, 117203 (2007).

${ }^{43}$ F. Elste and C. Timm, Phys. Rev. B 75, 195341 (2007). 
${ }^{44}$ J. Fernández-Rossier and R. Aguado, Phys. Rev. Lett. 98, 106805 (2007).

${ }^{45}$ J. Fransson, Phys. Rev. B 77, 205316 (2008).

${ }^{46}$ A. S. Núñez and R. A. Duine, Phys. Rev. B 77, 054401 (2008).

${ }^{47}$ L. Michalak, C. M. Canali, and V. G. Benza, Phys. Rev. Lett. 97, 096804 (2006).

${ }^{48}$ M. Misiorny and J. Barnaś, Phys. Rev. B 76, 054448 (2007).

${ }^{49}$ M. A. Barral, R. Weht, G. Lozano, and A. M. Llois, Physica B 398, 369 (2007).

${ }^{50}$ J. Merino and O. Gunnarsson, Phys. Rev. B 69, 115404 (2004).

${ }^{51}$ C. Cohen-Tannoudji, G. Grynberg, and J. Dupont-Roc, AtomPhoton Interactions (Wiley, New York, 1998).

${ }^{52}$ C. Le Gall, L. Besombes, H. Boukari, R. Kolodka, J. Cibert, and H. Mariette, Phys. Rev. Lett. 102, 127402 (2009).

${ }^{53} \mathrm{C}$. Hirjibehedin (private communication).
${ }^{54}$ N. Lorente and J.-P. Gauyacq, Phys. Rev. Lett. 103, 176601 (2009).

${ }^{55}$ B. Sothmann and J. König, New J. Phys. 12, 083028 (2010).

${ }^{56}$ A. N. Rudenko, V. V. Mazurenko, V. I. Anisimov, and A. I. Lichtenstein, Phys. Rev. B 79, 144418 (2009).

${ }^{57} \mathrm{C}$. Lin and B. Jones, arXiv:1003.4841 (unpublished).

${ }^{58}$ M. Persson, Phys. Rev. Lett. 103, 050801 (2009).

${ }^{59}$ J. van Bree, P. M. Koenraad, and J. Fernández-Rossier, Phys. Rev. B 78, 165414 (2008).

${ }^{60}$ J.-P. Gauyacq, F. D. Novaes, and N. Lorente, Phys. Rev. B 81, 165423 (2010).

${ }^{61}$ E. B. Myers, D. C. Ralph, J. A. Katine, R. N. Louie, and R. A. Buhrman, Science 285, 867 (1999).

${ }^{62}$ F. D. Novaes, N. Lorente, and J.-P. Gauyacq, Phys. Rev. B 82, 155401 (2010). 